\title{
Evolution of massive AGB stars
}

\section{III. the thermally pulsing super-AGB phase ${ }^{\star}$}

\author{
L. Siess ${ }^{1,2}$ \\ 1 Institut d'Astronomie et d'Astrophysique, Université Libre de Bruxelles, ULB, CP 226, 1050 Brussels, Belgium \\ e-mail: siess@astro.ulb.ac.be \\ 2 Centre for Stellar and Planetary Astrophysics, School of Mathematical Sciences, Monash University, Victoria 3800, Australia
}

Received 27 October 2009 / Accepted 15 December 2009

ABSTRACT

\begin{abstract}
Aims. We present the first simulations of the full evolution of super-AGB stars through the entire thermally pulsing AGB phase. We analyse their structural and evolutionary properties and determine the first SAGB yields.

Methods. Stellar models of various initial masses and metallicities were computed using standard physical assumptions which prevents the third dredge-up from occurring. A postprocessing nucleosynthesis code was used to compute the SAGB yields, to quantify the effect of the third dredge-up (3DUP), and to assess the uncertainties associated with the treatment of convection.

Results. Owing to their massive oxygen-neon core, SAGB stars suffer weak thermal pulses, have very short interpulse periods and develop very high temperatures at the base of their convective envelope (up to $140 \times 10^{8} \mathrm{~K}$ ), leading to very efficient hot bottom burning. SAGB stars are consequently heavy manufacturers of ${ }^{4} \mathrm{He},{ }^{13} \mathrm{C}$, and ${ }^{14} \mathrm{~N}$. They are also able to inject significant amounts of ${ }^{7} \mathrm{Li},{ }^{17} \mathrm{O},{ }^{25} \mathrm{Mg}$, and ${ }^{26,27} \mathrm{Al}$ in the interstellar medium. The 3DUP mainly affects the CNO yields, especially in the lower metallicity models. Our post-processing simulations also indicate that changes in the temperature at the base of the convective envelope, which would result from a change in the efficiency of convective energy transport, have a dramatic impact on the yields and represent another major source of uncertainty.
\end{abstract}

Key words. stars: AGB and post-AGB - stars: evolution - nuclear reactions, nucleosynthesis, abundances - stars: abundances

\section{Introduction}

In the past decade, new SAGB models have been computed that address specific aspects of their peculiar evolution, such as the propagation of the carbon-burning flame (e.g. Garcia-Berro et al. 1997; Siess 2006), the effects of overshooting (Gil-Pons et al. 2007), semiconvection (Poelarends et al. 2008), or thermohaline mixing (Siess 2009). The start of electron captures reactions in the oxygen-neon $(\mathrm{ONe})$ core and the URCA process have also been investigated (Ritossa et al. 1999), and non-solar models were computed and their main properties analysed (e.g. Gil-Pons et al. 2005; Siess 2007). But none of these works have thoroughly study the thermally pulsing super-AGB (TP-SAGB) phase or released yields for these stars.

Although the main structural features of their evolution seems to be reasonably well understood, their fate is still highly uncertain. The main reasons are ascribed to our poor knowledge of the mass loss rate and third-dredge-up properties that control how much mass can be removed during the post-carbon burning evolution (Gil-Pons et al. 2007; Siess 2007; Poelarends et al. 2008). If the envelope is lost before electron capture reactions are activated in the core, an ONe white dwarf forms, otherwise the core collapses and a neutron star forms. The critical core mass corresponding to this evolutionary bifurcation is $\sim 1.37 \mathrm{M}_{\odot}$ (Nomoto 1984).

Available stellar models also show a large scatter in the mass range of SAGB stars, which by definition, is bracketed be-

* Tables 2 to 6 are only available in electronic form at the CDS via anonymous ftp to cdsarc.u-strasbg.fr (130.79.128.5) or via http://cdsweb.u-strasbg. fr/cgi-bin/qcat?]/A+A/512/A10 tween $M_{\text {up }}$ (the minimum mass for carbon ignition) and $M_{\text {mas }}$ (the minimum mass above which the star proceeds through all nuclear-burning stages up to the formation of an iron core). The differences between these simulations come from the treatment of mixing and the difficulty of current models for determining the extent of the convective core, especially during the central helium-burning phase. For example, applying a moderate overshooting at the edge of the convective core shifts the entire SAGB mass range down by $2 M_{\odot}$ (Gil-Pons et al. 2007; Siess 2007). Our conservative treatment of convective boundaries (application of the strict Schwarzschild criterion and absence of numerical diffusion at the convective boundaries) provides an upper limit to the SAGB stars, which in our case range between 8 and $11 M_{\odot}$.

Despite the uncertainties affecting the determination of $M_{\text {up }}$ and $M_{\text {mas }}$, standard IMFs still predict a large number of SAGB stars. Indeed, according to the Salpeter IMF, there are as many stars in the mass range 7-11 as massive stars with $M>11 M_{\odot}$. But despite their importance, they have been neglected in galactic chemical evolution models mainly because of the lack of stellar yields. This absence of data is largely caused by the appalling amount of CPU time that is required to follow their full evolution. As discussed in this paper, the omission of this large stellar population may have significant effects on the galactic evolution of ${ }^{7} \mathrm{Li},{ }^{13} \mathrm{C}$, and ${ }^{14} \mathrm{~N}$.

The goal of this paper is to provide the first yields for SAGB stars and describe some properties of their TP-SAGB evolution. The paper is organised as follows: in the next section, the main physical ingredients of the stellar evolution code and some general model properties are presented. Then in Sect. 3 
we describe the structural evolution during the thermally pulsing phase. Section 4 analyses the nucleosynthesis, both in the pulse and in the envelope. The yields are derived in Sect. 5 along with uncertainties. The implications of SAGB for the chemical evolution of globular clusters is mentioned in Sect. 6, and the paper ends with a general discussion.

\section{The models}

The models presented in this paper are computed with the same version of STAREVOL as described in Siess (2007) and with the following main physical assumptions: Vassiliadis \& Wood (1993) mass loss rate with no metallicity dependence, the Schwarzschild criterion to delineate the convective boundaries, and the absence of extra-mixing beyond the convective boundaries that, in our case, prevents the third dredge-up and use of a constant mixing length parameter $\alpha=1.75$. These models do not include core overshooting. Details about the network and nuclear reaction rates can be found in Siess \& Arnould (2008). The initial composition is scaled to solar according to the Grevesse et al. (1996) mixture. Concerning the treatment of mixing, we assume instantaneous mixing of all chemical species in the convective zones. In these regions, the nucleosynthesis is computed in the one-zone approximation with mass-averaged reaction rates. As shown in Siess \& Arnould (2008), this treatment remains a good approximation but prevents the accurate determination of the ${ }^{7} \mathrm{Li}$ abundance.

Computation of SAGB stars requires specific rezoning strategies, during both the flame propagation (Siess 2006) and the interpulse phase where a high spatial resolution is needed at the base of the convective envelope. In this very thin region of a few $10^{-5} M_{\odot}$ (see Sect. 3.1 and Fig. 3), the main variables show very steep gradients, and rezoning is quite intense, inevitably generating some numerical noise. In addition to constantly re-adapting the grid points to the moving H-burning shell, the composition of the envelope is also homogenised in each model, bringing protons where they should not really be present. Although the one-zone approximation used to treat the nucleosynthesis in the convective zone gives the right energy budget ${ }^{1}$, it introduces small oscillations in the nuclear energy production which reflects in an irregular behaviour of $T_{\text {env }}$ as illustrated in Fig. 2. Increasing the spatial resolution at the base of the envelope or the constraints on the timestep reduces this numerical artifact, but the effects are always very small (variations in $T_{\text {env }}$ never exceed $<2.5 \%$ ).

The calculation of SAGB models is extremely timeconsuming. A typical model comprises 1200-2500 numerical shells, the number increasing during the thermal pulse. During the interpulse period, the time step is primarily constrained by $\mathrm{H}$ burning and by the requirement that no more than $5 \%$ of protons are depleted in any shell between two consecutive models. Because all SAGB stars undergo very efficient hot bottom burning (HBB) with temperatures in the H-burning shell (HBS) as high as $1.5 \times 10^{8} \mathrm{~K}$, the time step becomes very short, of the order of $\sim 10^{-3}-10^{-2} \mathrm{yr}$ during the interpulse. As a consequence, more than $3 \times 10^{7}$ time steps were required in some simulations, representing more than 6 months CPU on a powerful desktop. It should also be emphasised that all the models presented in this paper fail to converge near the tip of the SAGB, mostly because of the density inversion in the surface layers of the star but this

\footnotetext{
1 This was checked by running test simulations where mixing and nucleosynthesis were coupled.
}

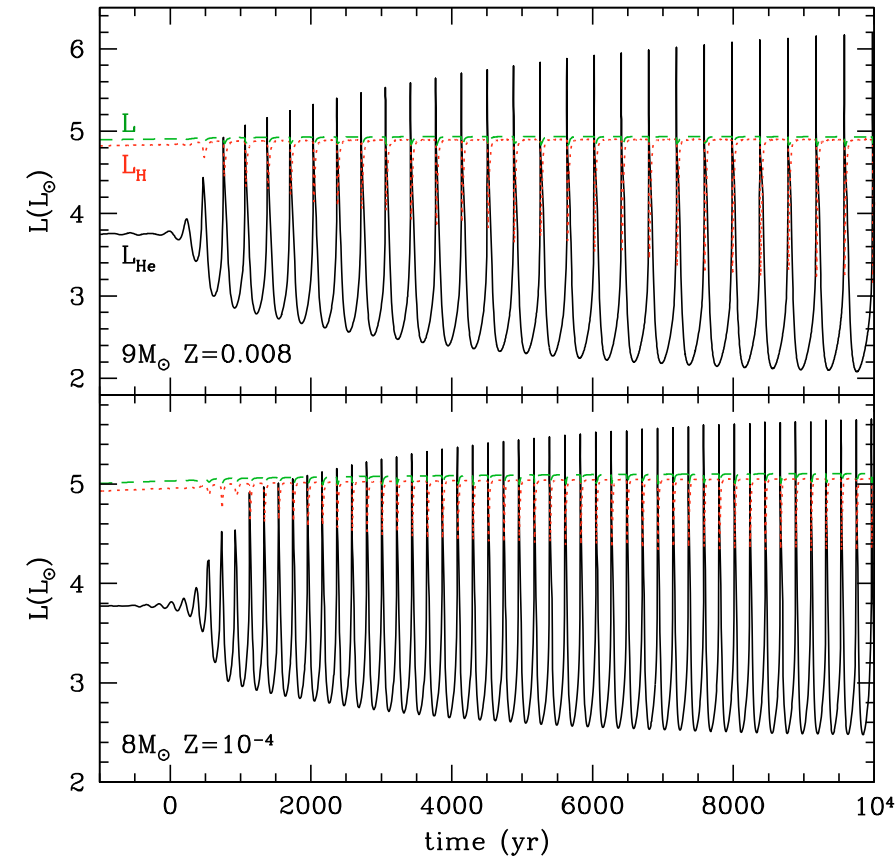

Fig. 1. Evolution of the $\mathrm{He}\left(L_{\mathrm{He}}\right.$, solid line), $\mathrm{H}\left(L_{\mathrm{H}}\right.$, dotted line) and surface ( $L$, dashed line just above the $L_{\mathrm{H}}$ curve) luminosities at the beginning of the TP-SAGB phase of a $9 M_{\odot}, Z=0.008$ (top panel) and $8 M_{\odot}, Z=10^{-4}$ (lower panel) stellar model. The origin of time has been reset to the time when the first He-shell instability develops.

is a well known (and unsolved!) problem that may be associated with $\mathrm{H}$ recombination (Wagenhuber \& Weiss 1994).

\section{The thermally pulsing super-AGB phase}

We resume the evolution where it was stopped at the end of Siess (2007), after the completion of the second dredge-up when carbon burning is powering off. Similarly to their lower mass counterparts, our stellar models enter an early-SAGB phase during which the He-burning shell (HeBS) becomes thinner and gets closer to the HBS as the core contracts. The nuclear energy production is progressively transferred from the HeBS to the HBS, which now supplies more than $80 \%$ of the stellar luminosity. At that stage, the conditions (Schwarzschild \& Härm 1965; Yoon et al. 2004) are met for the development of recurrent thermal instabilities in the HeBS and the star enters the TP-SAGB (Fig. 1). It is worth noticing that, following the second dredge-up and up to the first pulse, the temperature at the base of the convective envelope increases significantly from a few $10^{7} \mathrm{~K}$ up to $\sim 100-140 \times 10^{6} \mathrm{~K}$.

Figure 2 illustrates a typical pulse-interpulse cycle in a $10.5 M_{\odot}, Z=0.02$ model. The structural evolution is similar to that of a lower mass AGB star with alternate $\mathrm{H} / \mathrm{He}$-burning phases. However, because of the relative weakness of the thermal pulses ( $L_{\mathrm{He}} \lesssim 10^{6} L_{\odot}$ in this model), the surface luminosity is almost unaffected by the He-shell instability. Most of the energy released by the pulse is absorbed in the layers located at the base of the convective envelope, and the expansion produces a partial extinction of the H-burning shell (in Fig. $2 L_{\mathrm{H}}>10^{3} L_{\odot}$ ). After the decay of the He-shell instability, the star enters the quiescent interpulse phase where efficient H-burning extends into the convective envelope and provides more than $85 \%$ of the surface luminosity. Along the AGB, the star suffers intense mass 


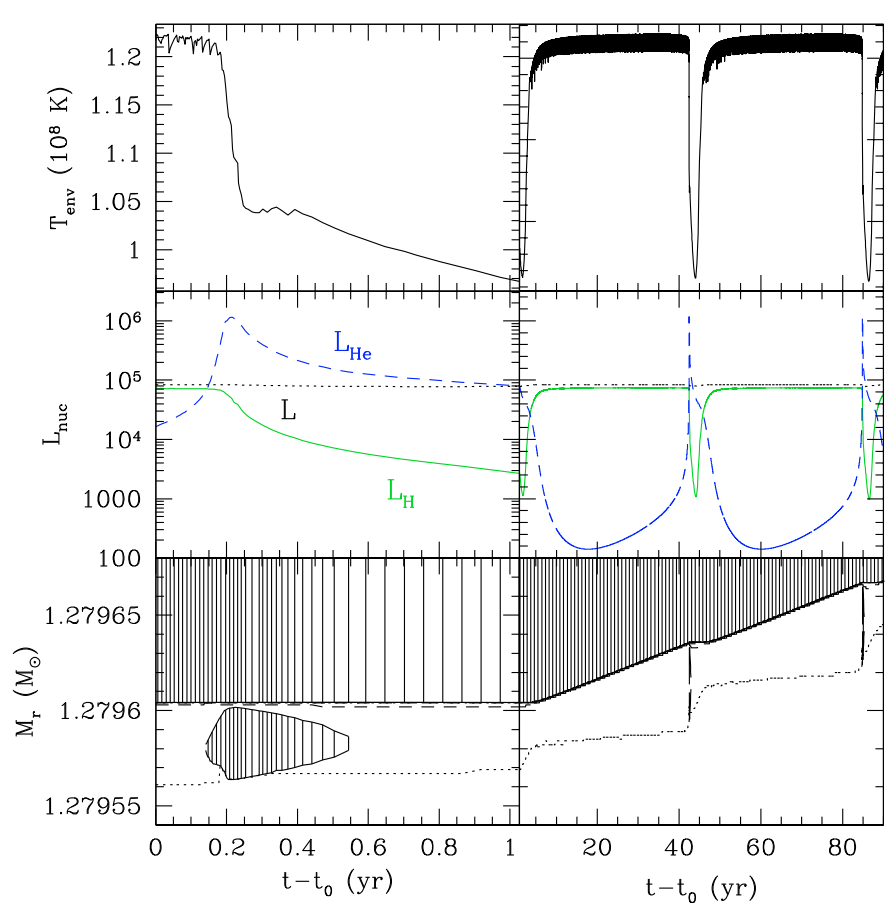

Fig. 2. Structural evolution of a $10.5 M_{\odot}, Z=0.02$ model during a pulse-interpulse cycle. The evolution of the temperature at the base of the envelope ( $\left.T_{\text {env }}\right)$ and of the He (dashed), $\mathrm{H}$ (solid), and surface luminosity (dotted) are shown in the top and mid panels, respectively. The bottom panel is a Kippenhahn diagram, and the origin of time was artificially reset to better illustrate the timescales.

loss with values up to a few $10^{-4} M_{\odot} \mathrm{yr}^{-1}$. With the progressive expulsion of the envelope, both the surface luminosity and mass loss rate decline, progressively revealing the core.

\subsection{Structure of TP-SAGB stars}

The structure of an SAGB star is very similar to that of a "standard" AGB star except for the mass and composition of the core. Low- and intermediate-mass stars avoid carbon burning and enter the AGB phase with a $\mathrm{CO}$ core of typically $0.5-0.7 M_{\odot}$. In contrast, SAGB stars ignite carbon off centre and build up a massive $\mathrm{ONe}$ core $\left(M_{\mathrm{ONe}} \gtrsim 1.05 M_{\odot}\right.$, Siess 2007). Because the interior is degenerate, the ONe core is also more compact $\left(R_{\text {core }} \propto M_{\text {core }}^{-1 / 3}\right)$, which leads to a much stronger gravitational pull at the base of the convective envelope. As a consequence, the layers surrounding the core are more compressed and thus much thinner and hotter. This results in SAGB stars being more luminous and more extended than their lower mass counterparts. The typical interpulse structure of an $8.5 M_{\odot}, Z=0.004$ model is presented in Fig. 3. Primarily made of ${ }^{16} \mathrm{O}(\sim 60 \%)$ and ${ }^{20} \mathrm{Ne}$ $(\sim 30 \%)$, the degenerate core is almost isothermal. The nuclear energy production is confined to a very narrow region of a few $10^{-5} M_{\odot}$ in the vicinity of envelope base where the density, temperature, and pressure profiles drop abruptly by several orders of magnitude. The thin region where the nuclear energy is produced extends over a few pressure scale heights inside the convective envelope, leading to hot bottom burning.

\subsection{The thermal pulse properties}

After the second dredge-up (2DUP), $\mathrm{H}$ re-ignites and when enough helium has accumulated in the intershell, the $\mathrm{He}$
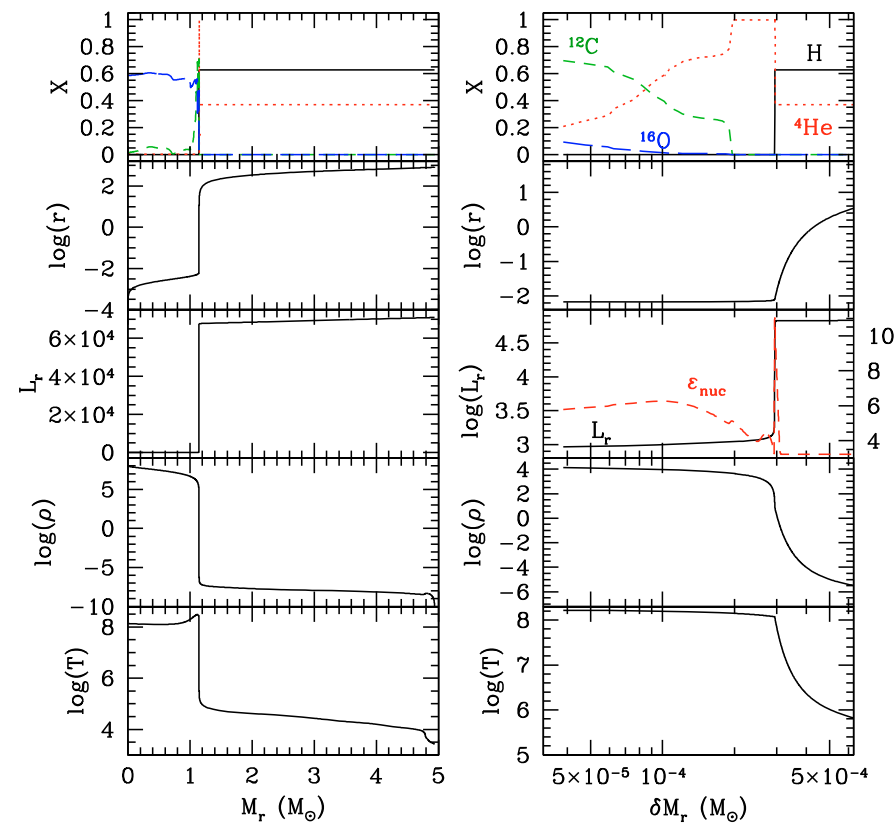

Fig. 3. Internal structure of an $8.5 M_{\odot}, Z=0.004$ model during an interpulse. From top to bottom the different panels represent the H (blacksolid), ${ }^{4} \mathrm{He}$ (red-dotted), ${ }^{12} \mathrm{C}$ (green-short-dashed) and ${ }^{16} \mathrm{O}$ (blue-longdashed) chemical profiles (in mass fraction), the radius ( $\mathrm{r}$ ), the luminosity $\left(L_{r}\right)$, density $(\rho)$, and temperature profiles $(T)$, respectively. The right panel is a zoom of the region located at the base of the convective envelope where $\delta M_{r}=M_{r}-1.1448 M_{\odot}$. The logarithm of the nuclear energy production (dashed line right $y$-abscissa) is also plotted in the mid right panel along with $L_{r}$. The base of the convective envelope is located at $M_{r}=1.145095 M_{\odot}$.

luminosity starts to oscillate. The first instabilities are weak $\left(L_{\mathrm{He}}<L\right)$ and rapidly evolve into fully developed convective thermal pulses.

As shown in Fig. 4, the strength of the He-shell flash $\left(L_{\mathrm{He}}\right)$ initially increases rapidly as the mass extent of the convective pulse $\left(\Delta M_{\text {pulse }}\right)$ grows. After a few thousand years, core contraction has significantly slowed down, the slope of the $r_{\text {core }}$ curves becomes much shallower, and $\Delta M_{\text {pulse }}$ reaches an extremum. The stabilisation of the core also marks the transition to an asymptotic regime where the main properties of the SAGB star are now evolving more slowly. In this regime, $L_{\mathrm{He}}$ continues to increase but at a modest rate and $\Delta M_{\text {pulse }}$ slowly declines as the core grows. For a given composition, the mass of the convective instability thus decreases with age and with increasing initial mass. Quantitatively, $\Delta M_{\text {pulse }}$ varies between $10^{-5}$ and $3 \times 10^{-3} M_{\odot}$ for $M$ decreasing from 10.5 to $7.5 M_{\odot}$. This is 2 to 3 orders of magnitude smaller than in a $3 M_{\odot}$ AGB stars.

SAGB are also characterised by the occurrence of weak thermal pulses. To understand the origin of this behaviour, we compare in Fig. 5 the profiles of relevant quantities in the pulse region of a 3 and $10 M_{\odot}$ at different epochs during the development of the instability. From this plot we see that in the SAGB the temperature is higher but the degeneracy $(\eta)$ and the ratio of gas pressure to total pressure $(\beta)$ are smaller compared to AGB stars. These conditions contribute to making the thermal instability weaker in SAGB stars (Yoon et al. 2004). Indeed with a lower degeneracy and larger $\beta$, the pressure response to a temperature perturbation is faster and stronger $\left(P_{\text {rad }} \propto T^{4}\right)$ so less energy can accumulate. Besides, when the temperature is higher, the dependence of the nuclear reaction rate on $T$ is less (parameter $v$ in Yoon et al. 2004) so the runaway is less 


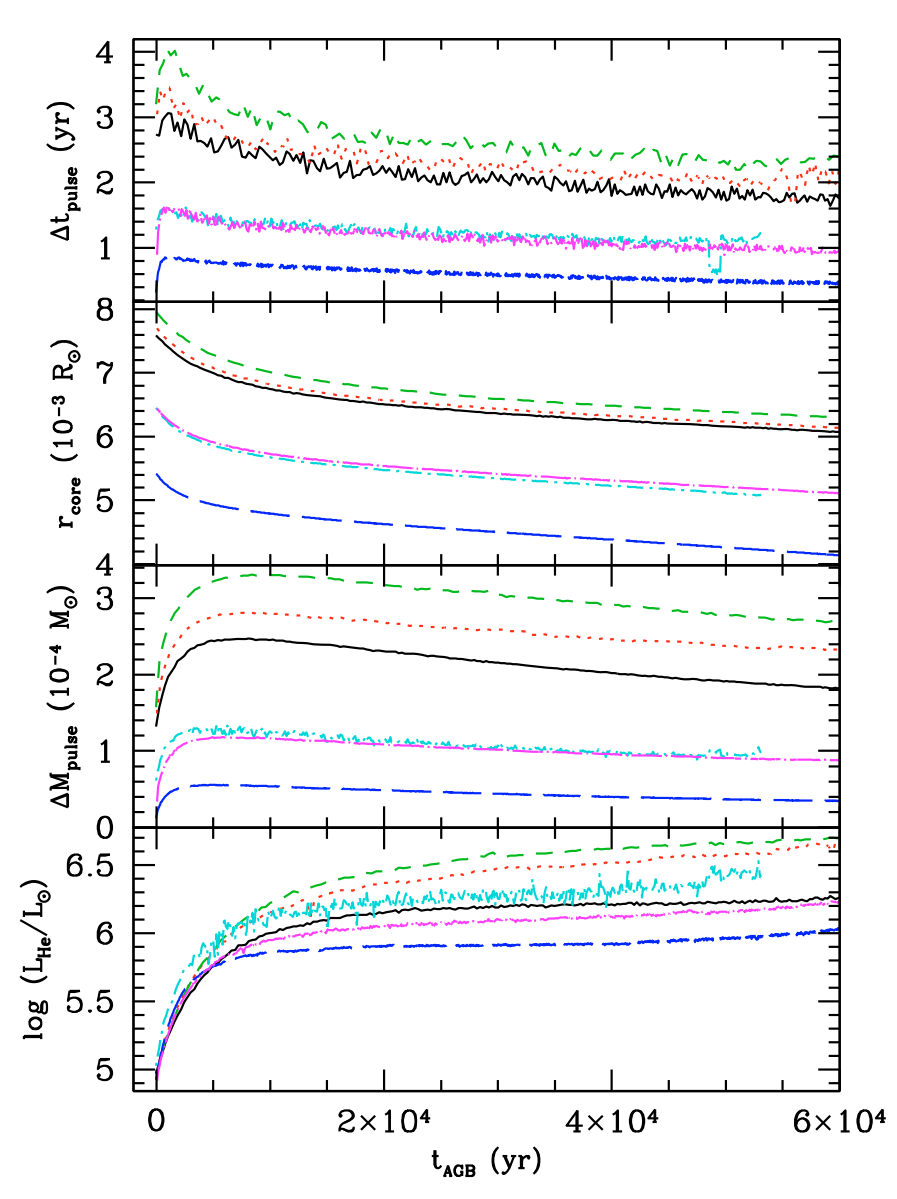

Fig. 4. Evolution of the pulse duration $\left(\Delta t_{\text {pulse }}\right)$, core radius $\left(r_{\text {core }}\right)$, pulse mass $\left(\Delta M_{\text {pulse }}\right)$, and maximum He-shell luminosity $\left(L_{\mathrm{He}}\right)$ for selected TP-SAGB models. The origin of time coincides with the occurrence of the first thermal pulse. With increasing $L_{\mathrm{He}}$, the models correspond to (mass, $Z$, colour $)=(9,0.001$, blue $),(8.5,0.001$, magenta $),(8,0.001$, black), (10, 0.02, cyan), (8.5, 0.004, red), and (9, 0.008, green).

violent. All these factors concur to abbreviate and weaken the thermal pulse provided the conditions for shell instability are met, in particular the relative thinness of the HeBS (parameter $\alpha_{\mathrm{s}}$ in Yoon et al. 2004). Although the HeBS is thinner in SAGB stars $\left(\Delta r_{\mathrm{HeBS}}=2.6 \times 10^{-3} R_{\odot}\right.$ in our $10 M_{\odot}$ star compared to $3.79 \times 10^{-2} R_{\odot}$ in the $3 M_{\odot}$ model $)$, it is also located at a smaller radius $\left(6.61 \times 10^{-3}\right.$ vs. $\left.3.13 \times 10^{-2} R_{\odot}\right)$. The result is that $\alpha_{\mathrm{s}}$ is very similar between these models so the shell is not any stabler in SAGB stars.

The pulse duration ( $\left.\Delta t_{\text {pulse }}\right)$ is weakly dependent on the initial stellar composition. It is primarily determined by the core mass and steadily decreases during the evolution. To a reasonably good approximation, we find that

$\Delta t_{\text {pulse }}=\left(189.3-3.15 M_{\mathrm{c}}\right) \exp \left(-1.31 M_{\mathrm{c}}\right)+40.42 \mathrm{yr}$,

where $M_{\mathrm{c}}$ is the core mass in $M_{\odot}$. This leads to pulse durations of at most a few years. It is important to stress that the duration of the pulse appears to be almost independent of $L_{\mathrm{He}}$.

The highest temperature reached at the base of the pulse $\left(T_{\text {pulse }}\right)$ increases during the TP-SAGB phase and reaches $3.7 \times$ $10^{8} \mathrm{~K}$ in the most massive models. Such temperatures may have some interesting nucleosynthetic implications for the s-process (see Sect. 4.1). For a given core mass, $T_{\text {pulse }}$ is an increasing function of metallicity. The strength of the pulse depends on the temperature in the HeBS, so it is stronger in stars of the same

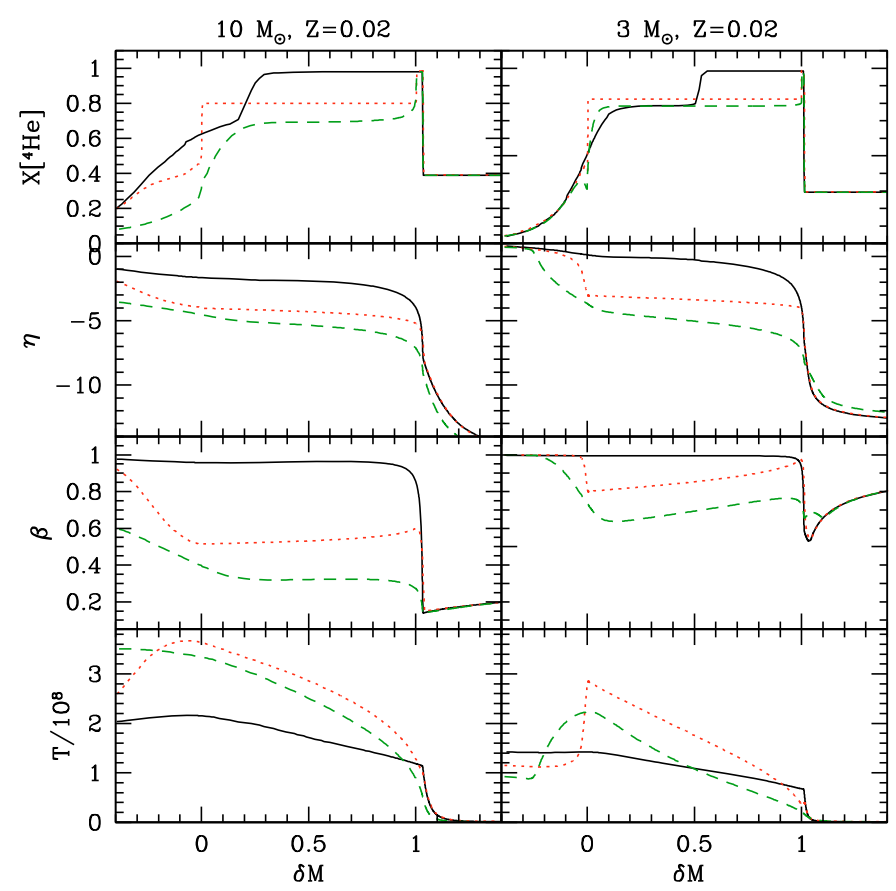

Fig. 5. Physical conditions in the He-burning shell during a thermal pulse in a $10 M_{\odot}$ (left) and $3 M_{\odot}$ (right) $Z=0.02$ models. The temperature $(\mathrm{T})$, the ratio of the gas to the total pressure $(\beta)$, degeneracy $(\eta)$, and the ${ }^{4} \mathrm{He}$ mass fraction are shown as a function of the relative mass coordinate $\delta M=\left(M_{r}-M_{\text {base }}^{\text {pulse }}\right) / \Delta M_{\text {pulse }}$ at the beginning (solid line), at the peak luminosity (dotted line), and just after the disappearance of the convective instability (dashed line). In the 10 and $3 M_{\odot}$ models, $\Delta M_{\text {pulse }}$ equals $10^{-5}$ and $1.11 \times 10^{-2} M_{\odot}$, respectively.

core mass with higher $Z$. It should also be recalled that stars with a higher initial metal content develop smaller convective cores during central $\mathrm{H}$ and $\mathrm{He}$ burning and start their TP-SAGB phase at a lower core mass (e.g. Siess 2007). Consequently, for a given core mass, a more metal-rich star has a more massive envelope which imposes a stronger compressional heating at the base of the He burning shell.

The overlap factor $r_{\text {over }}=\Delta M_{\text {over }} / \Delta M_{\text {pulse }}$, where $\Delta M_{\text {over }}$ is the mass of the previous pulse that is engulfed in the next pulse of mass $\Delta M_{\text {pulse }}$, increases rapidly until the star reaches its asymptotic regime and then decreases slowly. In the asymptotic regime, there is a good, almost linear, anti-correlation between $r_{\text {over }}$ and the pulse strength $L_{\mathrm{He}}$. In stars with a higher core mass (i.e. $L_{\mathrm{He}}$ ), the overlap is smaller and $r_{\text {over }}$ increases with increasing pulse mass. The overlap factor is quite important for the s-process nucleosynthesis because it controls the amount of s-process elements that can be exposed to multiple irradiations in the convective pulse. However, the magnitude and evolution of $r_{\text {over }}$ strongly depend on the 3DUP efficiency.

\subsection{The interpulse phase}

After the decay of the thermal pulse, the HBS reignites and the star enters the interpulse phase. In the asymptotic regime, the relation between the interpulse $\left(\Delta t_{\text {inter }}\right)$ and pulse $\left(\Delta t_{\text {pulse }}\right)$ durations is given, to a good approximation, by

$\log \left(\Delta t_{\text {pulse }}\right)=0.79 \log \left(\Delta t_{\text {inter }}\right)-1.59$

where $\Delta t_{\text {pulse }}$ and $\Delta t_{\text {inter }}$ are expressed in years. 
The minimum HeBS luminosity during the interpulse phase $\left(L_{\mathrm{He}, \mathrm{min}}\right)$ is strongly anti-correlated with the pulse luminosity $L_{\mathrm{He}}$, and to very good accuracy we have the following relation

$\log \left(L_{\mathrm{He}}\right)=-1.427 \log \left(L_{\mathrm{He}, \min }\right)+9.1542$.

Therefore, the weaker the He burning during the interpulse, the stronger the subsequent flash (see also Mowlavi 1995). During the ascent of the AGB phase, $L_{\mathrm{He} \text {,min }}$ decreases reflecting a more efficient cooling of the HeBS consecutive to a larger expansion caused by a stronger pulse.

The intershell mass is also a decreasing function of time, the consequence of the increasing gravitational pull of the growing core. The $\mathrm{H}$ and He burning shells are thus getting thinner and closer to each other. The mass accreted on the He-buffer during the interpulse $\Delta M_{\mathrm{H}}$ varies between $10^{-5}-5 \times 10^{-4} M_{\odot}$ in our considered models, which is 2 orders of magnitude lower than in a typical $3 M_{\odot}$ star where $\Delta M_{\mathrm{H}} \approx 4-5 \times 10^{-3} M_{\odot}$.

At the beginning of the TP-SAGB phase, the temperature at the base of the convective envelope ( $\left.T_{\text {env }}\right)$ increases rapidly as a result of a strong core contraction subsequent to the quenching of $\mathrm{C}$ burning. When the star enters its full amplitude regime, $T_{\text {env }}$ starts to decline slowly with the reduction of the envelope mass $\left(M_{\text {env }}\right)$. Near the end of the evolution, when $M_{\text {env }} \lesssim 2-3 M_{\odot}$, $T_{\text {env }}$ drops more abruptly, bringing hot bottom burning to a stop. Generally, stars of a given core mass develop higher envelope temperatures when the metallicity decreases because, at the time the comparison is made, more metal poor stars have a more massive envelope, but this conclusion is somewhat dependent on the assumed mass loss rate.

After a rapid increase at the beginning of the TP-SAGB phase, the core growth rate $\dot{M}_{\text {core }}$ remains almost constant despite a continuous decline in $T_{\text {env }}$. Apparently the reduction of the nuclear-burning efficiency is compensated by the decline in the surface luminosity so the variation of $\dot{M}_{\text {core }}$ is small. For the mass and metallicity ranges covered by our models, we find $5 \times 10^{-7} M_{\odot} \mathrm{yr}^{-1} \leq \dot{M}_{\text {core }} \leq 8 \times 10^{-7} M_{\odot} \mathrm{yr}^{-1}$. The core growth rate increases with core mass but there is no obvious dependence on $T_{\text {env }}$. For a given composition, more massive stars also have higher $\dot{M}_{\text {core }}$. We note, however, a strong anti-correlation between this quantity and the radius of the bottom of the convective envelope, reflecting the direct influence of the gravitational pull.

The activation of $\mathrm{HBB}$ produces the break down of the classical Paczyński (1970) core mass-luminosity $\left(M_{\mathrm{c}}-L\right)$ relation (Bloecker \& Schoenberner 1991; Lattanzio 1992; Bloecker 1995). As shown by Refsdal \& Weigert (1970) and Tuchman et al. (1983), the existence of an $M_{\mathrm{c}}-L$ relation requires an inert transition region below the convective envelope where the luminosity remains constant and where the temperature, pressure, density, and radius present a steep dependence on the mass coordinate. In stars experiencing $\mathrm{HBB}$, this transition region is absent because $\mathrm{H}$ burning extends into the convective envelope. In this circumstance, the properties of the core cannot be decoupled from those of the envelope. The additional production of nuclear energy generated at the base of the envelope makes the star more luminous and departs significantly from the classical $M_{\mathrm{c}}-L$ relation as illustrated in the bottom panel of Fig. 6. The surface luminosity reaches a peak value when $T_{\text {env }}$ is also at its maximum and then declines during most of the TP-SAGB evolution as the envelope mass shrinks. Eventually HBB stops and a classical $M_{\mathrm{c}}-L$ relation can be recovered. Similar behaviour was also reported by Marigo et al. (1998) in their massive $\left(M \geq 4 M_{\odot}\right)$ AGB models.

The core mass at the first thermal pulse $\left(M_{\mathrm{c}}^{1 \mathrm{TP}}\right.$ is defined when $L_{\mathrm{He}}$ first exceeds $L_{\mathrm{H}}$ ) is an important quantity that gives

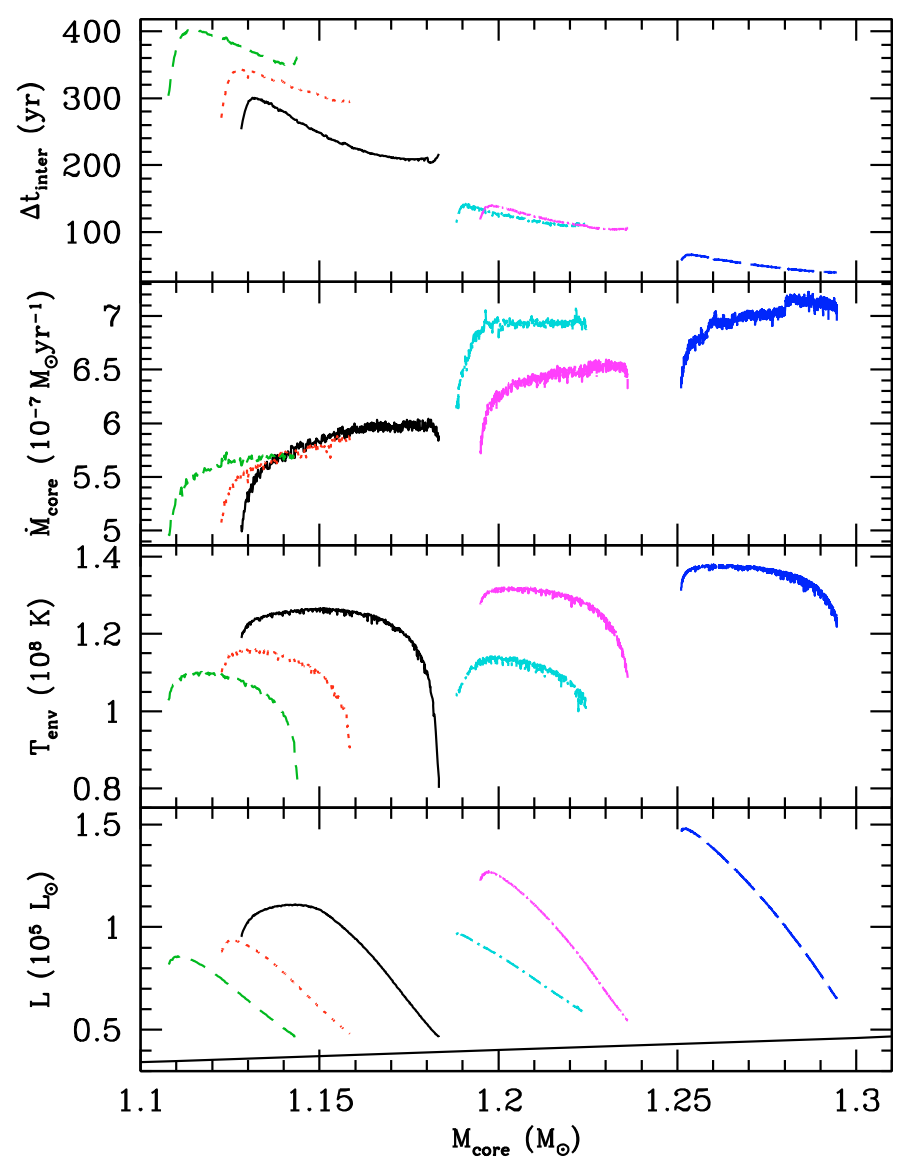

Fig. 6. Evolution of the interpulse period $\left(\Delta t_{\text {inter }}\right)$, core growth rate ( $\left.\dot{M}_{\text {core }}\right)$, temperature at the base of the convective envelope $\left(T_{\text {env }}\right)$, and surface luminosity as a function of core mass for selected computed models. The initial core mass corresponds to $M_{c, 0}$. With increasing core mass, the models correspond to (mass, $Z$, colour $)=(9,0.008$, green), (8.5, 0.004, red), $(8,0.001$, black), $(10,0.02$, cyan $),(8.5,0.001$, magenta) and $(9,0.001$,blue). The solid line in the lower panel represents the classical Paczyński (1970) $M_{\mathrm{c}}-L$ relation.

a lower limit to the remnant mass and is intimately related to the initial/final mass relation. For a given initial mass, $M_{\mathrm{c}}^{1 \mathrm{TP}}$ steadily increases with decreasing metallicity and for a given metallicity $M_{\mathrm{c}}^{1 \mathrm{TP}}$ increases quite linearly with initial stellar mass but the slope of the $M_{\mathrm{c}}^{1 \mathrm{TP}}(M)$ curve is slightly steeper than the one given by Wagenhuber \& Groenewegen (1998).

The properties of our $10 M_{\odot}, Z=0.02$ star compare quite well with the model computed by Ritossa et al. (1996): the core mass $\left(1.21 M_{\odot}\right.$ vs. $1.188 M_{\odot}$ in this work), pulse duration $(1.5 \mathrm{yr}$ vs. $1.23 \mathrm{yr})$, pulse mass $\left(1.34 \times 10^{-4} M_{\odot}\right.$ Vs. $\left.1.5 \times 10^{-4} M_{\odot}\right)$ and temperature $\left(3.69 \times 10^{8} \mathrm{~K}\right.$ vs. $\left.3.6 \times 10^{8} \mathrm{~K}\right)$, as well as the envelope temperature $\left(\sim 1-1.1 \times 10^{8} \mathrm{~K}\right)$ and overlap factor $(0.23 \mathrm{vs}$. $0.25)$ are very similar. Our core growth rate $\left(6.9 \times 10^{-7} M_{\odot} \mathrm{yr}^{-1}\right.$ vs. $\left.5.7 \times 10^{-7} M_{\odot} \mathrm{yr}^{-1}\right)$ is, however, slightly higher and the interpulse duration consequently shorter in our models (120 yr vs. $200 \mathrm{yr}$ ). The origin of these differences is hard to assess given the many differences between these simulations in terms of both the constitutive physics (EOS, opacities, nuclear rates), physical prescriptions (MLT, mass loss rate, etc.) and numerics. At the beginning of the TP-SAGB phase, the envelope composition of the $10 M_{\odot}$ model of Ritossa et al. (1996) is slightly different for ours because of the different initial composition (they start with $Y=0.28$ and $Z=0.02$ while we use $Y=0.27$ and $Z=0.018$ ) 
Table 1. General properties of the TP-SAGB models.

\begin{tabular}{|c|c|c|c|c|c|c|c|c|c|c|c|c|c|c|c|c|c|c|}
\hline $\begin{array}{r}M_{\text {ini }} \\
M_{\odot}\end{array}$ & Z & $\begin{array}{c}M_{c}^{1 \mathrm{TP}} \\
M_{\odot}\end{array}$ & $\begin{array}{c}M^{1 \mathrm{TP}} \\
M_{\odot}\end{array}$ & $\begin{array}{c}T_{\mathrm{env}}^{1 \mathrm{TP}} \\
10^{8} \mathrm{~K}\end{array}$ & $\begin{array}{c}\Delta t_{\text {pulse }} \\
\mathrm{yr}\end{array}$ & $\begin{array}{c}L_{\mathrm{He}}^{\max } \\
10^{6} L_{\odot}\end{array}$ & $\begin{array}{l}\Delta M_{\text {pulse }}^{\max } \\
10^{-4} M_{\odot}\end{array}$ & $\begin{array}{c}T_{\text {pulse }}^{\max } \\
10^{8} \mathrm{~K}\end{array}$ & $r_{\text {over }}$ & $\begin{array}{r}t_{\text {inter }} \\
\text { yr }\end{array}$ & $\begin{array}{c}M_{\text {core }} \\
M_{\odot} \mathrm{yr}^{-1}\end{array}$ & $\begin{array}{c}M_{\text {core }}^{\text {end }} \\
M_{\odot}\end{array}$ & $\begin{array}{c}\overline{T_{\text {env }}} \\
10^{8} \mathrm{~K}\end{array}$ & $\begin{array}{c}T_{\mathrm{env}}^{\max } \\
10^{8} \mathrm{~K}\end{array}$ & $\begin{array}{r}\Delta t_{\text {env }} \\
\text { yr }\end{array}$ & $\begin{array}{c}t_{\text {SAGB }} \\
10^{6} \mathrm{yr}\end{array}$ & $\begin{array}{r}\Delta t_{\mathrm{SAGB}} \\
\mathrm{yr}\end{array}$ & $n_{\mathrm{TP}}$ \\
\hline $7.5^{\dagger}$ & 0.0001 & 11.069 & 7.497 & 1.201 & 1.10 & 0.345 & 3.687 & 3.095 & 112 & 116.46 & $6.04(-7)$ & 1.284 & 1.392 & 1.549 & 223330 & 37.6603 & 356374 & 2776 \\
\hline 8. & & 142 & & & 80 & & & & & & & & & & & & & \\
\hline & & & & & 66 & & & & & & & & 1.385 & & & & & \\
\hline 9. & & 11 & 991 & & 0.45 & & 78 & 569 & & & & & 1.317 & & & & & \\
\hline 8. & & $\begin{array}{ll}0 & 1.128\end{array}$ & 980 & 190 & 1.90 & 752 & 2.4 & 494 & & & & & 1.054 & & 003 & & & 394 \\
\hline & & 195 & .460 & 1.277 & 1.13 & 231 & & 3.5 & & & & & 1.030 & & & & & 546 \\
\hline 9.0 & & 251 & .973 & 1.312 & 0.58 & .830 & 56 & 3.609 & 8 & & & & 1.080 & & & & 909 & 1237 \\
\hline 7.0 & & 981 & 77 & & & & & 2 & & & & & & & & & & 139 \\
\hline & & & & & & & & 3.4 & & & & & & & & & & 165 \\
\hline 8. & & & & & & & & & & & & & & & & & & 201 \\
\hline 9. & & & & & & & & & & & & & & & & & & 01 \\
\hline 9. & & & & & & & & & & & & & & & & & & 115 \\
\hline 10. & & & & & & & & & & & & & & & & & & 03 \\
\hline & & & & & & & & & & & & & & & & & & 109 \\
\hline & & & & & & & & & & & & & & & & & & 173 \\
\hline 9. & & & & & & & & & & & & & & & & & & 291 \\
\hline $10 .($ & & & & & & & & & & & & & 0.986 & & & & & 1043 \\
\hline & & & & & & & & & & & & & & & & & & 119 \\
\hline 9. & & & & & & & & & & & & & 0.656 & & & & & 209 \\
\hline 10.0 & & & & & & & & 690 & & 12 & & & 0.681 & & & & & 437 \\
\hline 10. & & & & & & & & & & & & & 0.9 & & & & & 1090 \\
\hline 9. & & & & & & & & & & & & & 0.4 & & & & & 98 \\
\hline 9.5 & & $\begin{array}{lll}0 & 1.109\end{array}$ & 8.922 & 0.902 & 2.26 & 1.543 & 2.768 & 3.370 & 0.271 & 71.14 & & 1.191 & 0.534 & 0.975 & 63440 & & 123625 & 180 \\
\hline
\end{tabular}

Notes. ${ }^{\dagger}$ These models do not ignite carbon.

The description of the variables is given at the end of Sect. 3.3.

and because of the depth of the 2DUP, which allows a larger helium enrichment in their model ( $Y=0.397$ vs. 0.387 in our models). Either way, these abundances will be largely reshaped during the thermally pulsing SAGB phase, especially since the Ritossa et al. (1996) model experiences 3DUP.

We present in Table 1 selected properties of the evolution of our SAGB models. The first and second columns identify the star by its initial mass and metallicity, Cols. 3-5 present the core mass $\left(M_{\mathrm{c}}^{1 \mathrm{TP}}\right)$, stellar mass $\left(M^{1 \mathrm{TP}}\right)$ and envelope temperature $\left(T_{\text {env }}^{1 \mathrm{TP}}\right)$ at the first thermal, Cols. 6-10 give the average pulse duration $\left(\Delta t_{\text {pulse }}\right)$, the highest HeBS luminosity $\left(L_{\mathrm{He}}^{\max }\right)$, the maximum extent of the He-driven convective zone $\left(\Delta M_{\text {pulse }}^{\max }\right)$, the maximum temperature at the base of the pulse $\left(T_{\text {pulse }}^{\max }\right)$ and the mean overlap factor $\left(r_{\text {over }}\right)$, Cols. $11-13$ present the average interpulse duration $\left(\Delta t_{\text {inter }}\right)$, mean core growth rate $\left(\dot{M}_{\text {core }}\right)$ and final core mass $\left(M_{\text {core }}^{\text {end }}\right)$, and Cols. $14-19$ the mean envelope temperature defined as $\overline{T_{\text {env }}}=\int T_{\text {env }} \mathrm{d} t$ where the integral starts from the time of the first thermal pulse, maximum envelope temperature, as well as the period $\left(\Delta t_{\text {env }}\right)$ during which $T_{\text {env }}>\overline{T_{\text {env }}}$, the stellar age at the first thermal pulse $\left(t_{\mathrm{SAGB}}\right)$, the duration $\left(\Delta t_{\mathrm{SAGB}}\right)$ of the TP-SAGB phase (time started from the first thermal pulse), and the number of computed thermal pulses $\left(n_{\mathrm{TP}}\right)$. This number has to be compared with the total number of pulses that are estimated from our postprocessing calculations and specified in Tables 2-6 (only available at the CDS) by $n_{\text {pul }}$.

\section{Nucleosynthesis and surface abundance evolution}

At the end of the main sequence, SAGB stars with metallicities $Z>0.001$ experience the 1DUP. The main chemical signature is a surface enrichment in ${ }^{14} \mathrm{~N}$ and ${ }^{13} \mathrm{C}$ concomitant with a decrease in ${ }^{12} \mathrm{C}$ and ${ }^{15} \mathrm{~N}$ resulting in a ${ }^{12} \mathrm{C} /{ }^{13} \mathrm{C}$ ratio of $\sim 20$
(Siess 2007). As far as the CNO are concerned, the signatures of the 2DUP are very similar to those of the 1DUP but because convection is able to penetrate deeper into the interior, the products of the $\mathrm{NeNa}$ and $\mathrm{MgAl}$ chains are also mixed in the envelope. As a result, the 2DUP is responsible for a very strong envelope enrichment in helium and, to a lower extend, of ${ }^{23} \mathrm{Na}$. The dredgeout phenomenon, which occurs in the most massive SAGB stars at the end of carbon burning (Iben et al. 1997; Siess 2006), also increases the envelope abundances of ${ }^{4} \mathrm{He}$ and ${ }^{12} \mathrm{C}$ significantly and at the end if this event the star becomes $\mathrm{C}$-rich $(\mathrm{C} / \mathrm{O}>1)$.

In the next section, we describe the nucleosynthesis taking place in the pulse and analyse how the composition inherited from the first and second dredge-ups is modified during the TP-SAGB phase.

\subsection{Pulse composition and nucleosynthesis}

The energetics of the pulse is driven by He burning and mainly leads to the production of ${ }^{12} \mathrm{C}$ and ${ }^{16} \mathrm{O}$. However, the conversion of helium is incomplete and at the time of the 3DUP, the typical intershell composition is ${ }^{4} \mathrm{He}:{ }^{12} \mathrm{C}:{ }^{16} \mathrm{O}=0.66: 0.31: 0.02$. The intershell composition, and primarily the abundances of ${ }^{12} \mathrm{C}$ and ${ }^{16} \mathrm{O}$, depends on the pulse temperature and on the treatment of mixing. For instance, Boothroyd \& Sackmann (1988) find in their standard AGB models ${ }^{4} \mathrm{He}:{ }^{12} \mathrm{C}:{ }^{16} \mathrm{O}=0.76: 0.22: 0.02$ in agreement with our values. Herwig et al. (1997), who included in their simulations some overshooting below the pulse, find a substantially different composition with ${ }^{4} \mathrm{He}:{ }^{12} \mathrm{C}:{ }^{16} \mathrm{O}=0.25: 0.50: 0.25$.

The pulse ingests large amounts of ${ }^{14} \mathrm{~N}$, which was produced by $\mathrm{HBB}$, and converts it into ${ }^{22} \mathrm{Ne}$ after successive $\alpha$-captures through the reactions ${ }^{14} \mathrm{~N}(\alpha, \gamma){ }^{18} \mathrm{O}(\alpha, \gamma)$. The high temperature reached at the base of the convective pulse $\left(T_{\text {pulse }} \gtrsim 3.2 \times 10^{8} \mathrm{~K}\right)$ allows an efficient burning of ${ }^{22} \mathrm{Ne}$ via the $(\alpha, \mathrm{n})$ and $(\alpha, \gamma)$ 

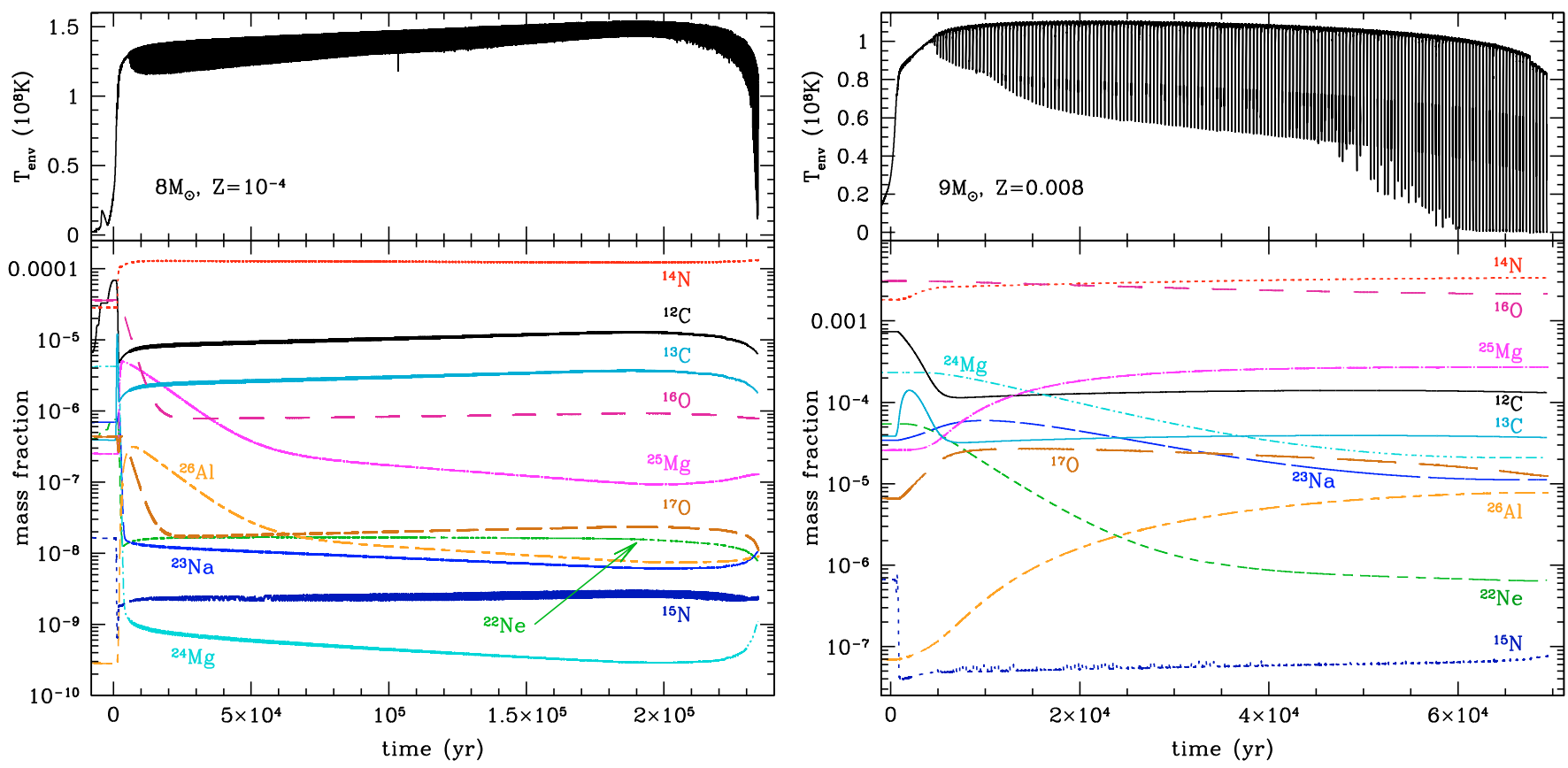

Fig. 7. Evolution of $T_{\text {env }}$, the temperature at the base of the convective envelope (top panels) and of the surface mass fractions of selected nuclei along the TP-SAGB of an $8 M_{\odot}, Z=10^{-4}$ (right) and $9 M_{\odot}, Z=0.008$ (left). The origin of time has been reset to the time when $T_{\text {env }}=3 \times 10^{7} \mathrm{~K}$.

channels leading to the synthesis of ${ }^{25,26} \mathrm{Mg}$. Above $T \gtrsim 3.3 \times$ $10^{8} \mathrm{~K}$, the ${ }^{22} \mathrm{Ne}(\alpha, \mathrm{n})$ reaction becomes an important neutron source, and neutron densities as high as $10^{11} \mathrm{~cm}^{-3}$ can be achieved in the hottest pulses. The released neutrons participate into the nucleosynthesis of heavy s-elements but also contribute to the production of ${ }^{17} \mathrm{O}$ and ${ }^{23} \mathrm{Na}$ after $(\mathrm{n}, \gamma)$ reactions on ${ }^{16} \mathrm{O}$ and ${ }^{22} \mathrm{Ne}$, respectively. Note that ${ }^{17} \mathrm{O}$ and ${ }^{13} \mathrm{C}$ reach equilibrium abundances between their production by $(n, \gamma)$ reactions on ${ }^{12} \mathrm{C}$ and ${ }^{16} \mathrm{O}$, respectively, and their destruction by $(\alpha, \mathrm{n})$ reactions. ${ }^{14} \mathrm{~N}$ is an efficient neutron poison that leads to the production of protons via ${ }^{14} \mathrm{~N}(\mathrm{n}, \mathrm{p})$ reactions. The released protons then participates into the fluorine production through the following chain of reactions ${ }^{18} \mathrm{O}(\mathrm{p}, \alpha){ }^{15} \mathrm{~N}(\alpha, \gamma){ }^{19} \mathrm{~F}$. Not all the ${ }^{15} \mathrm{~N}$ is produced by the previous reaction as some was present in the HBS. Above $T \gtrsim 2.8 \times 10^{8} \mathrm{~K}$, fluorine is destroyed by ${ }^{19} \mathrm{~F}(\alpha, \mathrm{p})^{22} \mathrm{Ne}$, so its production is very weak in SAGB stars.

At the end of the short-lived thermal pulse, for $T_{\text {pulse }} \sim$ $3.5 \times 10^{8} \mathrm{~K}$, the neutron irradiation amounts to a $\tau \lesssim$ 0.01-0.02 mbarn ${ }^{-1}$. This is less than what is required from the weak s-process component occurring in the He-burning convective core of massive stars $\left(\tau \approx 0.06\right.$ mbarn $^{-1}$, Raiteri et al. 1991) and much less than what is expected from the radiative s-process nucleosynthesis operating during the interpulse of low and intermediate mass stars where the neutron source is provided by ${ }^{13} \mathrm{C}(\alpha, \mathrm{n})$ reactions and where $\tau \gtrsim 0.3 \mathrm{mbarn}^{-1}$. Because of this small neutron exposure, the production of s-process elements in the convective pulse is expected to be small, but this may be compensated for by the large number of irradiations. Additional studies are required to clarify this aspect.

\subsection{Evolution of the envelope abundances}

The absence of extra mixing at the edge of the convective boundaries prevents the development of 3DUP episodes in our simulations. Therefore, after the 2DUP, the evolution of the surface abundances is caused solely by the action of hot bottom burning, the efficiency of which is mainly governed by the temperature at the base of the convective envelope. To illustrate the main nucleosynthetic features of TP-SAGB stars, we base our analysis on two distinct and somewhat extreme stars showing large differences in the initial composition, pulse number, and envelope temperatures. Namely, we will discuss the case of a $8 M_{\odot}$, $Z=10^{-4}$ and a $9 M_{\odot}, Z=0.008$ where the evolution of the surface abundances of selected elements is reproduced in Fig. 7.

After a sharp increase in the temperature at the base of the convective envelope following the completion of the 2DUP, the $\mathrm{CNO}$ cycles are activated. ${ }^{12} \mathrm{C}$ is largely converted into ${ }^{14} \mathrm{~N}$, and the ${ }^{12} \mathrm{C} /{ }^{13} \mathrm{C}$ ratio reaches its equilibrium value close to $\sim 3-4$. ${ }^{15} \mathrm{~N}$ is also efficiently destroyed while ${ }^{17} \mathrm{O}$ is produced. Above $T_{\text {env }} \gtrsim 10^{8} \mathrm{~K},{ }^{16} \mathrm{O}$ is efficiently depleted by (p, $\gamma$ ) reactions, and in some hot models the $\mathrm{C} / \mathrm{O}$ ratio becomes more that unity. The star then becomes carbon rich, or more exactly, oxygen poor. We emphasise that this observational characteristic is not the result of a carbon enrichment but rather of efficient oxygen depletion.

When the temperature approaches $35 \times 10^{6} \mathrm{~K}$, protons get involved in the NeNa chain. ${ }^{22} \mathrm{Ne}$ is efficiently depleted to the benefit of ${ }^{23} \mathrm{Na}$. Below $90 \times 10^{6} \mathrm{~K},{ }^{23} \mathrm{Na}(\mathrm{p}, \alpha)$ is faster than ${ }^{23} \mathrm{Na}(\mathrm{p}, \gamma)$ and the NeNa-burning chain cycles, allowing equilibrium abundances to be reached among the involved nuclides. Above $90 \times 10^{6} \mathrm{~K}$, the channel to the $\mathrm{MgAl}$ chain opens and the efficient destruction of ${ }^{24} \mathrm{Mg}$ favours the production of the heavier magnesium isotopes as well as of ${ }^{26,27} \mathrm{Al}$. However, at the very high temperatures $\left(T_{\text {env }} \gtrsim 1.3 \times 10^{8} \mathrm{~K}\right)$ seen in our $8 M_{\odot}$, $Z=10^{-4}$ model, ${ }^{25} \mathrm{Mg}$ and ${ }^{26,27} \mathrm{Al}$ are also destroyed and ${ }^{28} \mathrm{Si}$ is produced. In short, very efficient $\mathrm{HBB}$ is responsible for a large production of ${ }^{14} \mathrm{~N},{ }^{17} \mathrm{O},{ }^{25} \mathrm{Mg}$ and ${ }^{26,27} \mathrm{Al}$. Note also that in the coolest stellar envelopes, such as the $9 M_{\odot}, Z=0.008$ shown in Fig. 7, the envelope is removed before substantial ${ }^{23} \mathrm{Na}$ depletion could occur.

It is worth emphasizing that HBB operates in an extremely thin region at the base of the convective envelope engulfing at most a few $10^{-5} M_{\odot}$. In this region where most of the luminosity is produced, the density drops from $\sim 10 \mathrm{~g} \mathrm{~cm}^{-3}$ down to $10^{-4} \mathrm{~g} \mathrm{~cm}^{-3}$ (Fig. 3). At the bottom of the envelope, the mixing 
timescale is shorter than the nuclear timescale associated with the main reactions (for details see Siess \& Arnould 2008), thus allowing fresh material from the upper part of the envelope to continuously feed the actively burning shells.

\section{SAGB yields}

Yields were computed using the post-processing code described in Siess \& Arnould (2008). This program inputs the results of the evolutionary calculations (in practice the run of $T_{\text {env }}$, luminosity, effective temperature and radius) and a representative envelope structure (taken during the interpulse) to recompute the evolution of the abundances in the convective zone. The equations of nucleosynthesis are solved simultaneously with the diffusion equation where the convective diffusion coefficient is given $D=1 / 3 v_{\text {conv }} \Lambda$ where $v_{\text {conv }}$ is the convective velocity provided by the MLT and $\Lambda=\alpha H_{P}$ the mixing length. This method reproduces the full computation quite accurately (to within $<10 \%$ ) and enables calculation of the ${ }^{7} \mathrm{Li}$ yields. This postprocessing code allows us to analyse the impact of nuclear reaction rates uncertainties and to assess the effects associated with the third dredge-up and with the treatment of convection. This program was also used to calculate the final TP-SAGB evolution, as all our simulations ended before the envelope was completely removed. In the final evolution towards the white dwarf, we assume that $T_{\text {env }}$ continues to decrease linearly with the envelope mass $\left(M_{\text {env }}\right)$ down to a temperature of $5 \times 10^{6} \mathrm{~K}$ when the envelope mass reaches $0.7 M_{\odot}$ and then remains constant. The exact final value of $T_{\text {env }}$ is not important as it is always below the minimum temperature for $\mathrm{HBB}$ activation. For the mass loss rate, we also consider a linear decrease in $\dot{M}_{\text {loss }}$ with $M_{\text {env }}$, the value of $\mathrm{d} \dot{M}_{\text {loss }} / \mathrm{d} M_{\text {env }}$ being estimated from the last computed models. These parametrisations reproduce the decrease in $T_{\text {env }}$ and $\dot{M}_{\text {loss }}$ found in the more evolved SAGB models computed by Doherty et al. (2010, in preparation).

\subsection{Sources of uncertainties}

Many aspects of stellar modelling may affect the determination of the stellar yields. Among them are the efficiency of the 3DUP, which remains highly uncertain and critically dependent on the assumed mixing processes operating at the base of the envelope. This situation is further complicated in the case of SAGB stars where nuclear burning is vigorous at the convective border. This is quite unfortunate because the 3DUP has a strong impact on the yields because it allows the products of the pulse nucleosynthesis to reach the envelope and eventually be expelled into the interstellar medium. In our post-processing calculations, we assumed a constant 3DUP efficiency $\lambda=0.8$ to maximise the effects and an intershell composition given by ${ }^{4} \mathrm{He}:{ }^{12} \mathrm{C}:{ }^{16} \mathrm{O}=0.66: 0.31: 0.02$. As mentioned in Siess \& Arnould (2008), the feedback of the 3DUP pollution on the evolutionary properties of the envelope is not taken into account in our postprocessing code. The results should thus be regarded as qualitative, especially at low metallicity where the modification of the envelope composition by the 3DUP can be large. A new grid of SAGB models experiencing 3DUP is currently running (Doherty et al. 2010, in prep.), and they will allow us to better quantify this effect.

Also crucial is the description of convection. As already shown by Sackmann \& Boothroyd (1991) and more recently by Ventura \& D'Antona (2005a), changing the mixing length parameter $\alpha$ or adopting a different formalism for convection can have a strong impact on the AGB evolution. In particular, when a higher value of $\alpha$ is adopted or when the full spectrum of turbulence (Canuto \& Mazzitelli 1991) is used, the stellar luminosity and the mass loss rate are higher. As a consequence the duration of the TP-SAGB phase is shorter and the pollution by the 3DUP episodes reduced. But changing $\alpha$ also modifies the thermal structure of the envelope and thus the efficiency of HBB. To simulate this effect (Siess \& Arnould 2008), we artificially scaled the temperature profile so that $T_{\text {env }}$ is increased or decreased by $\pm 10 \%$. This change in $T_{\text {env }}$ is also accompanied by a corresponding change in the mass loss rate so when HBB is more efficient, the mass loss rate is also increased because of the addition nuclear luminosity generated in the envelope. We arbitrarily increased $\dot{M}_{\text {loss }}$ by the same factor as $T_{\text {env }}$. We are aware of the limitations of this approach and emphasise again that these results should be taken with caution and not at face value.

The effects of nuclear reaction rates uncertainties are briefly discussed and the consequences of changing the mass loss rate also investigated. In the latter case, the feedback from changing the mass loss rate on the structure is not considered, so the conclusions of this analysis will again remain very qualitative. Nevertheless, such simulations provide us with a basic understanding on how the yields depend on these various parameters. We also emphasise that these effects are not independent and may act jointly.

\subsection{Yields results}

In this section, we define the yield $Y_{k}$ of species $k$ by

$Y_{k}=\frac{\int_{0}^{\tau} X_{k}(t) \dot{M}(t) \mathrm{d} t}{M_{k}(0)}$

where $\tau$ is the stellar lifetime, $\dot{M}$ the mass loss rate, $X_{k}$ the mass fraction of species $k$ and $M_{k}(0)$ its mass in the initial stellar model. A value of $Y_{k}>1$ thus means that over the entire evolution this element has been produced and the yield is said to be positive. Some values of $Y_{k}$ are shown in Fig. 9. But before starting our analysis, it should be kept in mind that the final yields strongly depend on the envelope composition at the beginning of the TP-SAGB, which was shaped by the previous dredge-ups.

Hydrogen is depleted during HBB and the higher $T_{\text {env }}$; i.e., the more massive and the more metal poor the star, the lower the $\mathrm{H}$ yield. By increasing the amount of ${ }^{12} \mathrm{C}$, the $3 \mathrm{DUP}$ also enhances $\mathrm{H}$ destruction by the $\mathrm{CN}$ cycle as its burning timescale is inversely proportional to the carbon abundance.

${ }^{3} \mathrm{He}$ is efficiently destroyed by HBB. The yields are negative and $Y_{\mathrm{He} 3}$ decreases with decreasing mass and metallicity. This is mainly a consequence of a longer TP-SAGB duration. Variation in $T_{\text {env }}$ has a weak impact on the ${ }^{3} \mathrm{He}$ yields because this element burns at much lower temperatures than those found at the envelope base of SAGB stars.

Following the 2DUP, the surface ${ }^{4} \mathrm{He}$ enrichment continues during the TP-SAGB phase and mass fractions as high as 0.4 may be reached in the envelope. It is interesting to note that the effects of 3DUP episodes are quite strong because of the combined effects of dredging-up He-rich $(Y \sim 0.66)$ intershell material and catalysing $\mathrm{H}$ destruction by enhancing the envelope ${ }^{12} \mathrm{C}$ mass fraction. The extreme enrichment seen in the $Z=10^{-4}$ models with 3DUP is clearly not realistic and stems from the limitation of our models, which do not take the feedback of these mixing episodes into account on the stellar structure. Nevertheless, the massive helium production, already present after the 2DUP, was invoked to explain the puzzling HB 
morphologies and multiple main sequences observed in some globular clusters (Pumo et al. 2008).

${ }^{7} \mathrm{Li}$ is produced during the TP-SAGB phase by the Cameron $\&$ Fowler (1971) mechanism and subsequently destroyed by ${ }^{7} \mathrm{Li}(\mathrm{p}, \alpha) \alpha$ when ${ }^{3} \mathrm{He}$ is no longer present in the envelope. The ${ }^{7} \mathrm{Li}$ production can be quite large given the mass of the envelope, and in the most massive and most metal-rich SAGB stars that experience the strongest winds, a substantial amount of ${ }^{7} \mathrm{Li}$ can be released in the interstellar medium before it is destroyed. Values of $\log \epsilon(\mathrm{Li})^{2}$ as high as 5 are achieved during the TP-SAGB phase. The ${ }^{7} \mathrm{Li}$ yield is positive in our higher mass SAGB models. In the lower metallicity stars, the mass loss rate is weaker, and when ${ }^{7} \mathrm{Li}$ has reached its maximum value and starts to burn, little mass has yet been ejected. This results in negative ${ }^{7} \mathrm{Li}$ yields for stars with $Z<10^{-3}$ and $M \lesssim 9$. As emphasised by Travaglio et al. (2001), the lithium yields strongly depend on the assumed mass loss rate. Although AGB stars are thought not to contribute significantly to the galactic Li content (Romano et al. $1999,2001)^{3}$, the exact implication of SAGB stars remains to be assessed by detailed chemical evolution models.

With increasing initial mass, the star begins its TP-SAGB phase with a higher envelope carbon abundance (see Table 5 of Siess 2007). In our standard models without 3DUP, the ${ }^{12} \mathrm{C}$ yields are negative, except in the most massive and metal poorest stars where the 2DUP enrichment cannot be erased by HBB. But the ${ }^{12} \mathrm{C}$ yields strongly depend on the efficiency of the 3DUP, especially in the lowest metallicity stars where its mass fraction in the envelope is initially very low. Simulating the 3DUP in the postprocessing calculations can increase the ${ }^{12} \mathrm{C}$ yields by more than one order of magnitude. The relative increase in the ${ }^{12} \mathrm{C}$ yields due to the 3DUP is greater in lower mass stars where $\mathrm{HBB}$ is less efficient despite the fact that these stars start their TP-SAGB evolution with a lower ${ }^{12} \mathrm{C}$ content. Accounting for possible variations in $T_{\text {env }}$ introduces additional uncertainties, but these effects are smaller than those associated with the 3DUP pollution. As expected, higher temperatures favour ${ }^{12} \mathrm{C}$ depletion. More metal-poor SAGB stars achieve higher $Y_{\mathrm{C} 12}$ values mainly because of the lower initial carbon abundance.

The high value of $T_{\text {env }}$ in SAGB stars forces the CN cycle to operate at equilibrium $\left({ }^{13} \mathrm{C} /{ }^{12} \mathrm{C} \sim 4\right)$ so the ${ }^{13} \mathrm{C}$ yields follow the same trend as the ${ }^{12} \mathrm{C}$ ones: they increase with increasing initial stellar mass and decreasing metallicity. The production of ${ }^{13} \mathrm{C}$ is substantial in SAGB stars, and the main uncertainties also arise from the action of the 3DUP, which further enhances its envelope abundance as a result of ${ }^{12} \mathrm{C}(\mathrm{p}, \gamma)$ reactions.

${ }^{14} \mathrm{~N}$ is massively produced in the TP-SAGB envelope by $\mathrm{HBB}$, and the dependence of $Y_{\mathrm{N} 14}$ on mass and composition are similar to those of ${ }^{13} \mathrm{C}$. Third dredge-up episodes increase the surface ${ }^{14} \mathrm{~N}$ abundance as a result of $\mathrm{CN}$ processing. This primary ${ }^{4}$ source of ${ }^{14} \mathrm{~N}$ becomes the dominant yield component, but it is also very dependent on the characteristics of the 3DUP (efficiency as well as intershell composition).

Interestingly, ${ }^{15} \mathrm{~N}$ and ${ }^{18} \mathrm{O}$ yields depend on the treatment of mixing as the nuclear timescale associated with (p, $\alpha)$ reactions become comparable to the convective turnover timescale. Using a simplified approach that neglects the coupling between convective transport and nuclear burning overestimates the ${ }^{15} \mathrm{~N}$ and

\footnotetext{
${ }^{2}$ By definition $\epsilon(\mathrm{Li})=\log (\mathrm{Li} / \mathrm{H})+12$ where the abundances of $\mathrm{Li}$ and $\mathrm{H}$ are by number.

3 Travaglio et al. (2001) reach a different conclusion, but their results are criticised by Ventura et al. (2002).

${ }^{4}$ Primary elements are those that are directly synthesised from $\mathrm{H}$ and $\mathrm{He}$ and that do not depend on the initial composition.
}

${ }^{18} \mathrm{O}$ destruction by more than one order of magnitude. The ${ }^{18} \mathrm{O}$ yields are always negative and the ${ }^{15} \mathrm{~N}$ yields are positive in the metal-poor stars with $Z \lesssim 0.004$. As a general trend, $Y_{\mathrm{N} 15}$ increases with the initial mass.

With $T_{\text {env }}$ exceeding $\sim 10^{8} \mathrm{~K}$, the $\mathrm{ON}$ cycle nearly operates at equilibrium and with increasing temperatures, ${ }^{16} \mathrm{O}$ is further converted into ${ }^{17} \mathrm{O}$ and ${ }^{14} \mathrm{~N}$. In the absence of 3DUP, the ${ }^{16} \mathrm{O}$ yields decrease with increasing mass and $T_{\text {env }}$ and with decreasing metallicity. The 3DUP feeds the envelope with ${ }^{16} \mathrm{O}$, and the enrichment is stronger at lower metallicity where the contrast between the envelope and dredged-up material compositions is more pronounced. Despite this primary injection from the intershell, $Y_{\mathrm{O} 16}$ remains negative in SAGB stars. ${ }^{17} \mathrm{O}$ is significantly produced by the ON cycle in SAGB stars. This represents another strong signature of efficient HBB.

Despite some modest production in the thermal pulse, ${ }^{19} \mathrm{~F}$ is subsequently destroyed by proton captures at the base of the hot convective envelope. ${ }^{19} \mathrm{~F}$ yields remain always negative.

The evolution of species involved in the $\mathrm{Ne}-\mathrm{Na}$ and $\mathrm{Mg}-\mathrm{Al}$ chains has been discussed in detail in Siess \& Arnould (2008) in the context of SAGB stars and we refer the reader to this publication for details. In short, given the very high temperatures encountered at the base of the SAGB envelope $\left(T_{\text {env }} \gtrsim 9 \times 10^{7} \mathrm{~K}\right)$, there is strong leakage into the $\mathrm{MgAl}$ chains favouring the production of ${ }^{25} \mathrm{Mg}$ and ${ }^{26,27} \mathrm{Al}$. In the hottest models, ${ }^{26} \mathrm{Mg}$ production is by-passed by ${ }^{26} \mathrm{Al}(\mathrm{p}, \gamma)^{27} \mathrm{Si}\left(\beta^{+}\right)^{27} \mathrm{Al}$. We also confirm the results of Karakas \& Lattanzio (2003) and Denissenkov \& Herwig (2003) that the production of heavy magnesium isotopes by HBB always leads to higher ${ }^{25} \mathrm{Mg}$. The 3DUP enriches the envelope with ${ }^{22} \mathrm{Ne}$ and ${ }^{25,26} \mathrm{Mg}$ and at low metallicity $\left(Z \lesssim 10^{-3}\right)$ the effects are even stronger than those induced by changing $T_{\text {env }}$. Overall, the yields of ${ }^{25} \mathrm{Mg}$ and ${ }^{26,27} \mathrm{Al}$ are positive, and the ${ }^{22} \mathrm{Ne},{ }^{23} \mathrm{Na}$, and ${ }^{24,26} \mathrm{Mg}$ ones negative. ${ }^{20} \mathrm{Ne}$ is almost unaltered while ${ }^{21} \mathrm{Ne}$ is efficiently burnt in the envelope.

To get a better feeling for how the 3DUP affects the surface abundances, we performed additional simulations where its efficiency was set to $\lambda=0.3$ (instead of 0.8 ), while keeping the same intershell composition. Decreasing the 3DUP efficiency reduces the amount of intershell material that is diluted in the envelope after each pulse and increases the core growth rate, which shortens the duration of the TP-SAGB phase. However, in realistic simulations this point may be wrong because of the feedback from the 3DUP pollution on the envelope structure and on the mass loss rate. In the framework of our model, we find the anticipated result that when $\lambda$ is smaller, the envelope enrichment in ${ }^{12} \mathrm{C}$ and its $\mathrm{CNO}$ by-products $\left({ }^{13} \mathrm{C},{ }^{14} \mathrm{~N},{ }^{17} \mathrm{O}\right)$ as well as ${ }^{22} \mathrm{Ne}$ and ${ }^{23} \mathrm{Na}$ is less. The yields of ${ }^{4} \mathrm{He},{ }^{7} \mathrm{Li}$, and of the $\mathrm{Mg}$ isotopes seem to be less affected by a change in $\lambda$. We also note that a modification of the 3DUP efficiency has a greater impact on the more metal-poor stars. In our simulations, the ${ }^{12,13} \mathrm{C}$ and ${ }^{14} \mathrm{~N}$ yields are reduced by a factor of $\sim 2$ when $\lambda$ decreases from 0.8 to 0.3 (see Tables 3 and 4 available at the CDS).

Using the Iliadis et al. (2001) reaction rates instead of the default Angulo et al. (1999) rates affects the production of the Ne, $\mathrm{Mg}$, and $\mathrm{Al}$ isotopes. The faster ${ }^{23} \mathrm{Na}(\mathrm{p}, \gamma)$ rate of Iliadis et al. (2001) favours the leakage into the $\mathrm{MgAl}$ chain and accentuates the sodium depletion. As mentioned in Siess \& Arnould (2008), the abundances of ${ }^{24} \mathrm{Mg}$ and ${ }^{26} \mathrm{Mg}$ are weakly affected by this change in nuclear reaction rates. But to correctly estimate the effects of the nuclear uncertainties, one should consider a more systematic approach like those developed by Iliadis et al. (2002), Stoesz \& Herwig (2003), or Izzard et al. (2007). Concerning the operation of the $\mathrm{NeNa}$ and $\mathrm{MgAl}$ chains, Izzard et al. (2007) show that in intermediate-mass stars experiencing strong HBB, 
the uncertainties on the nuclear cross section make the determination of the sodium and aluminium yields particularly unreliable. They report variations in ${ }^{26} \mathrm{Al}$ and ${ }^{23} \mathrm{Na}$ up to two orders of magnitude, of more than one dex for ${ }^{24} \mathrm{Mg}$ and ${ }^{27} \mathrm{Al}$, and by a factor between 2 and 7 for ${ }^{20} \mathrm{Ne}$ and ${ }^{21} \mathrm{Ne}$. However, in the case of SAGB stars, the nuclear uncertainties are generally much smaller because of the higher value of $T_{\text {env }}$ (Siess \& Arnould 2008). Of course, this does not remove all uncertainties, but at least they are expected to be smaller in our stars.

Increasing the mass loss rate has the first effect of reducing the duration of the TP-SAGB phase and the exposition of the envelope material to $\mathrm{HBB}$. As a consequence, more ${ }^{7} \mathrm{Li}$ and ${ }^{12,13} \mathrm{C}$ can survive $\mathrm{H}$ burning, so less ${ }^{4} \mathrm{He}$ and ${ }^{14} \mathrm{~N}$ are produced. Most of the elements involved in the $\mathrm{NeNa}$ and $\mathrm{MgAl}$ chains are also less exposed to nuclear burning and their yields are slightly higher when $\dot{M}_{\text {loss }}$ is higher. The ${ }^{25,26} \mathrm{Mg}$ enhancements are stronger at lower metallicity. But the mass loss rate also influences the thermal structure of the envelope and modifies the burning conditions at the base of the envelope, as well as the duration of the interpulse and the number of thermal pulses. Ventura \& D'Antona (2005b) show that increasing $\dot{M}_{\text {loss }}$ decreases the ${ }^{12} \mathrm{C}$ yields because in this case, their stellar models experience fewer 3DUPs. For the other elements and in particular for ${ }^{14} \mathrm{~N},{ }^{16} \mathrm{O}$ and ${ }^{23} \mathrm{Na}$, our synthetic simulations qualitatively agree with their self consistent models, producing the same abundance dependence on $\dot{M}_{\text {loss }}$.

Tables 2-6 presenting the yields are available in the electronic version of the paper and can also be retrieved from the author's web page ${ }^{5}$. Table 2 presents the standard yields, Tables 3 and 4 the yields computed with a 3DUP efficiency of $\lambda=0.8$ and 0.3 , and Tables 5 and 6 the yields computed by increasing and decreasing $T_{\text {env }}$ by $\pm 10 \%$, respectively. The number of thermal pulses experienced by the star $\left(n_{\text {pul }}\right)$ is specified in the third row. All the yields were computed using the postprocessing code considering the full coupling between the nuclear burning and the diffusive mixing.

\section{Implication for globular cluster evolution}

SAGB may also play an important role in the chemical evolution of GCs. These stellar clusters are characterised by C-N, Li-Na, $\mathrm{O}-\mathrm{Na}$, and $\mathrm{Mg}-\mathrm{Al}$ anticorrelations that are seen both in RGB and main sequence turn-off stars (for a review Gratton 2007). Stars within a given cluster show a remarkable homogeneity in the heavy elements abundances (Fe-group and $\alpha$-elements), along with an almost constant $\mathrm{C}+\mathrm{N}+\mathrm{O}$ abundance. These chemical peculiarities, which are not seen in field stars, can be explained in terms of hydrogen burning at high temperature (Kudryashov \& Tutukov 1988; Denissenkov \& Denissenkova 1990). Currently, the most plausible scenario for explaining these abundance patterns refers to the self-enrichment scenario in which an earlier stellar population contaminated the material out of which the presently observed stars formed. Fast-rotating massive stars (Decressin et al. 2007), as well as massive AGB stars undergoing efficient HBB (Ventura et al. 2001), have been identified as potential candidates. Both models have their pros and cons (e.g. Charbonnel 2007), and we will try to see in this section how the SAGB stars fit in this scenario. Our models have to comply with basically three main observational constraints: (i) the $\mathrm{C}+\mathrm{N}+\mathrm{O}$ should remain roughly constant (within a factor of 2); (ii) O-depletion is accompanied by Na-enhancement; and (iii) $\mathrm{Al}$ is largely produced. Putting aside the fast-rotating massive-star

5 http://www-astro.ulb.ac.be/ siess/

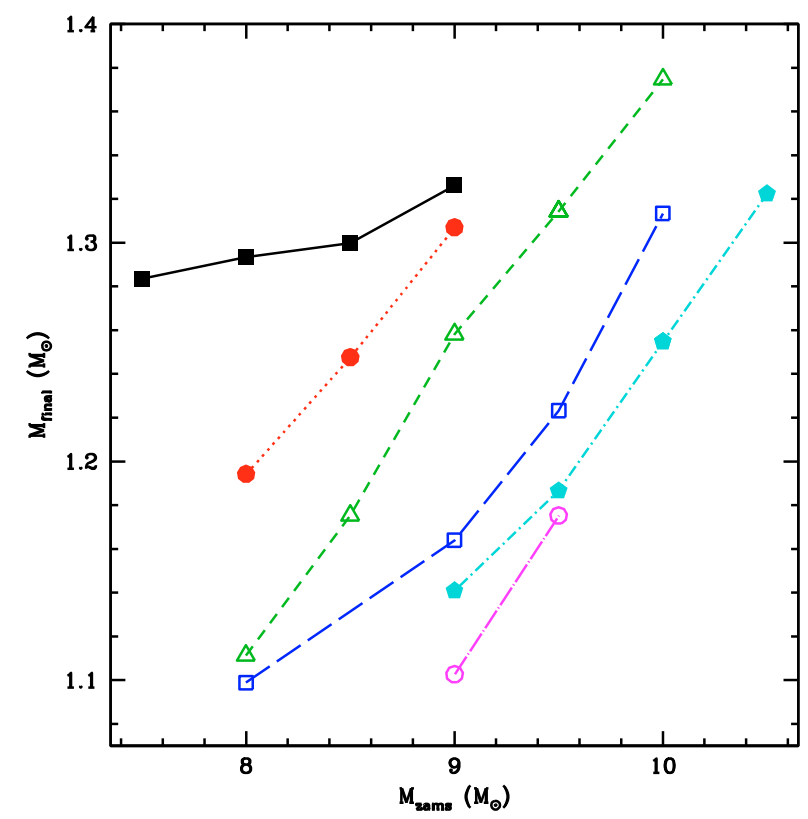

Fig. 8. Theoretical initial-final mass relation for the models computed with $Z=0.0001$ (black square), 0.001 (red filled circle), 0.004 (green open triangle), 0.008 (blue open square), 0.02 (cyan filled pentagon), and $Z=0.04$ (magenta open circle). As outlined in the text, $M_{\text {zams }}$ should be considered as an upper limit.

paradigm, only AGB stars in the narrow mass range 5-6 $M_{\odot}$ are able to achieve an encouraging agreement with the observations (Ventura \& D'Antona 2008) provided these stellar models include the highest cross section for the ${ }^{22} \mathrm{Ne}(\mathrm{p}, \gamma)$ reaction and an efficient convective energy transport (either by using the FST formulation for convection or a high value for the parameter $\alpha$ in the MLT). We stress, however, that if rotational mixing is included in the stellar models, this massive AGB stars scenario does not work. The reason is the transport of primary ${ }^{12} \mathrm{C}$ and ${ }^{16} \mathrm{O}$ by shear turbulence outside the convective core during central He burning. During the subsequent 2DUP the convective envelope swept through these layers and brings large amounts of carbon and oxygen to the surface, raising the $\mathrm{C}+\mathrm{N}+\mathrm{O}$ by up to 2 dex, in sharp contrast to current observations.

As far as the nucleosynthesis is concerned, SAGB models behave very much the same as massive AGB stars and thus have to face the same problems. In our standard non-rotating models, the absence of 3DUP maintains the sum $[(\mathrm{C}+\mathrm{N}+\mathrm{O}) / \mathrm{Fe}]$ constant, oxygen depletion can be very efficient (down to $[\mathrm{O} / \mathrm{Fe}]=$ $-1.8), \mathrm{Al}$ is produced ([Al/Fe] $\sim 0.4)$ if $T_{\text {env }}$ is not too high but the case of ${ }^{23} \mathrm{Na}$ is more problematic. ${ }^{23} \mathrm{Na}$ is efficiently depleted in the envelope, and the absence of 3DUP prevents its production by proton capture on the dredged-up ${ }^{22} \mathrm{Ne}$. Therefore, our more O-depleted models are also the most Na-poor. We are currently computing new SAGB models (Doherty et al. 2010, in prep.) where the 3DUP is activated. If the carbon enrichment is not too great, such that the $\mathrm{C}+\mathrm{N}+\mathrm{O}$ remains almost constant, we may be able to achieve a better agreement with the GC observations. These aspects will be addressed in detail in a forthcoming paper.

\section{Discussion and summary}

A direct output of our simulations is the first estimate of the theoretical initial-final mass relation for SAGB stars. The results (Fig. 8), however, remain largely uncertain given the 
L. Siess: Thermally pulsing super-AGB stars
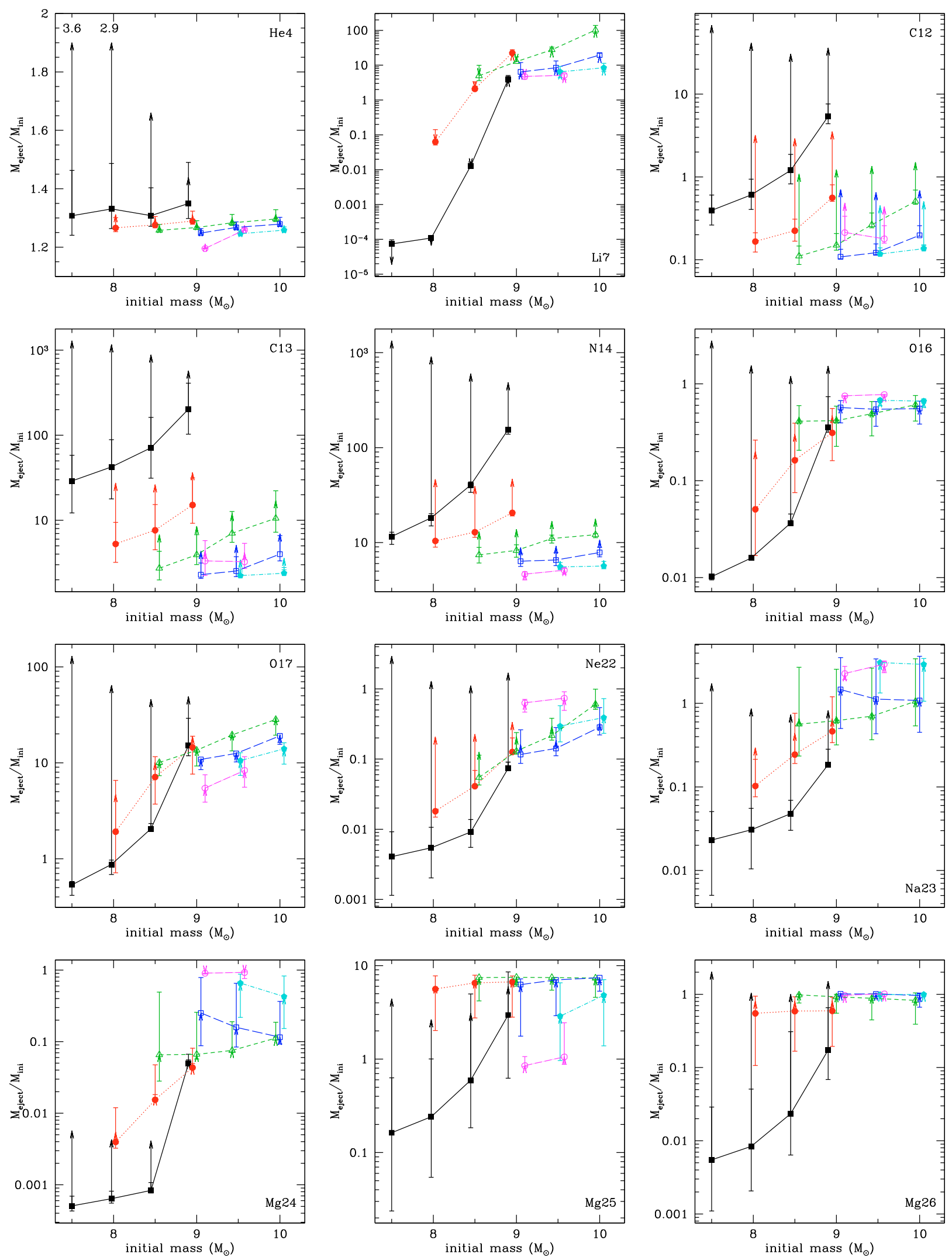

Fig. 9. Stellar yields $Y_{k}=M_{\text {eject }} / M_{\text {ini }}$ as a function of initial stellar mass for models with metallicity $Z=10^{-4}$ (black squares, solid lines), $Z=0.001$ (red filled circles, dotted lines), $Z=0.004$ (green open triangle, short-dashed lines), $Z=0.008$ (blue open squares, long-dashed lines), $Z=0.02$ (cyan pentagon, dot-dashed lines), and $Z=0.04$ (magenta open circle, short-long dashed lines). Error bars measure the uncertainties associated with a change in $T_{\text {env }}$ by $\pm 10 \%$. The arrow indicates the magnitude of the effects of the 3DUP, simulated with $\lambda=0.8$. 
overwhelming impact of core overshooting that can virtually shift the SAGB mass range down by $2 M_{\odot}$. We also emphasise that, with our conservative treatment of the convective boundaries (see Sect. 1), the initial SAGB mass should be regarded as an upper limit. The final mass $\left(M_{\text {fin }}\right)$ is also dependent on the mass loss and core growth rates (Siess 2007; Poelarends et al. 2008). For a given initial mass, $M_{\text {fin }}$ increases with decreasing metallicity because metal-poor stars develop a more massive convective core during central He burning. On the observational side, massive white dwarfs with $M_{\mathrm{WD}} \gtrsim 1 M_{\odot}$ have been observed (e.g. Należyty \& Madej 2004; Kepler et al. 2007), but determining the progenitor mass is difficult and highly uncertain. The observation of a peak in the WD mass distribution around 1.2 $M_{\odot}$ (Liebert et al. 2005) supports SAGB progenitors, although other channels involving accretion onto a $\mathrm{CO}$ white dwarf in a close binary evolution or binary mergers cannot be discarded. Furthermore, ONe WD are surrounded by an He-free shell and might be difficult to identify and to differentiate from a CO WD.

Throughout this study, convection appeared at the centre of many of our interrogations and was the source of many uncertainties. Because convection determines the temperature at the base of the envelope and fixes the extend of the stellar envelope, it directly affects the yields, as well as the mass loss rate, and in the end the fate of the SAGB star. As mentioned previously, all our models encounter converge problems near the tip of the SAGB because of a large density inversion in the ionisation layers. In a substantial fraction of the envelope radius, radiation pressure dominates and locally the adiabatic index $\Gamma_{1}$ falls below the critical value of 4/3. In addition or most likely as a consequence of these structural features, these evolved models display very high convective velocities $\left(v_{\text {conv }}\right)$. In the external layers of the envelope, $v_{\text {conv }}$ reaches $\approx 70-80 \%$ of the sound speed $c_{\mathrm{s}}$ and never gets lower than $0.4 c_{\mathrm{s}}$. The fact of using a constant $\alpha$ for the mixing length parameter, based on a solar calibration, is obviously not appropriate anymore. Hydrodynamical simulations of the envelope of giant stars (Freytag et al. 2002; Woodward et al. 2003; Brun \& Palacios 2009) have clearly established that many of the assumptions on which the MLT is based are violated, thus casting doubt on the reliability of this formalism in giant stars. Suspicions are also raised when trying to determine the location of the convective boundaries when active nuclear burning is operating at the base of the envelope. Our poor knowledge of convection presently limits our understanding of the structural and chemical evolution of SAGB stars. Only direct multidimensional simulations as courageously undertaken by Dearborn et al. (2006), Herwig et al. (2006), Meakin \& Arnett (2007), Palacios \& Brun (2008), Mocák et al. (2009) and others will eventually clarify these critical aspects.

According to the Salpeter IMF, SAGB stars are quite numerous so one would expect to see their chemical imprint on the galactic chemical evolution (GCE). In particular the large production of nitrogen and ${ }^{13} \mathrm{C}$ should show up after the extinction of the first SAGB population. Given that the SAGB lifetime is $\sim 3 \times 10^{7} \mathrm{yr}$, they are not expected to participate into the GCE before $[\mathrm{Fe} / \mathrm{H}] \simeq-3$. Chiappini et al. $(2005,2006)$ show that below that metallicity, fast-rotating massive stars can explain the large production of primary ${ }^{14} \mathrm{~N}$ observed in very metal-poor stars. However, her models have problems accounting for the high N/O ratios that are present around $\log (\mathrm{O} / \mathrm{H})+12 \sim 7$ (see Fig. 9 of Ekström et al. 2008), a region that corresponds precisely to the mass range of SAGB stars. This may be one of the signatures of their chemical pollution. But we also know that SAGB stars release substantial amounts of ${ }^{13} \mathrm{C}$. If correctly taken into account, their contribution may lower the ${ }^{12} \mathrm{C} /{ }^{13} \mathrm{C}$ ratio to the value observed in metal-poor stars (Chiappini et al. 2008). Consistent chemical evolution models that include realistic SAGB yields are needed to clarify their real impact on the GCE.

In summary, the evolution of thermally pulsing SAGB stars is very similar to that of intermediate mass stars. The main differences are the frequency and the strength of the instabilities, which are respectively much shorter and weaker than in their lower mass counterparts. Owing to their massive ONe cores, the internal structure of the TP-SAGB stars is hotter, favouring the ${ }^{22} \mathrm{Ne}(\alpha, \mathrm{n})$ reaction at the base of the pulse and very efficient HBB in the envelope. We showed that SAGB stars are heavy producers of ${ }^{14} \mathrm{~N},{ }^{13} \mathrm{C}$, as well as of ${ }^{17} \mathrm{O},{ }^{25} \mathrm{Mg},{ }^{26,27} \mathrm{Al}$ and in some cases of ${ }^{7} \mathrm{Li}$. They also produce a significant amount of ${ }^{4} \mathrm{He}$, and these signatures may have imprinted the early chemical evolution of galaxies and globular clusters. We emphasise that SAGB yields suffer large uncertainties, mainly from the treatment of convection, which determines the efficiency of $\mathrm{HBB}$ and the properties of the 3DUP, which strongly impact the CNO yields, especially in the most metal poor stars. In the near future, we will released new set of SAGB yields based on SAGB models that experience efficient 3DUPs (Doherty et al. 2010, in prep.). These new models with help evaluate the impact of SAGB stars on the chemical evolution of our galaxies and globular clusters.

Acknowledgements. L.S. wishes to express his deepest gratitude to John Lattanzio for providing him the opportunity to spend 5 months in a very stimulating and friendly scientific environment. He also thanks his colleagues from CSPA for their warm hospitality. This research was supported under Australian Research Council's Discovery Projects funding scheme (project number DP0877317), by the Communauté française de Belgique - Actions de Recherche Concertées, and by the FNRS. L.S. is an FNRS research associate.

\section{References}

Angulo, C., Arnould, M., Rayet, M., et al. 1999, Nucl. Phys. A, 656, 3 Bloecker, T. 1995, A\&A, 297, 727

Bloecker, T., \& Schoenberner, D. 1991, A\&A, 244, L43 Boothroyd, A. I., \& Sackmann, I.-J. 1988, ApJ, 328, 653 Brun, A. S., \& Palacios, A. 2009, ApJ, 702, 1078

Cameron, A. G. W., \& Fowler, W. A. 1971, ApJ, 164, 111

Canuto, V. M., \& Mazzitelli, I. 1991, ApJ, 370, 295

Charbonnel, C. 2007, in Why Galaxies Care About AGB Stars: Their Importance as Actors and Probes, ed. F. Kerschbaum, C. Charbonnel, \& R. F. Wing, ASP Conf. Ser., 378, 416

Chiappini, C., Matteucci, F., \& Ballero, S. K. 2005, A\&A, 437, 429 Chiappini, C., Hirschi, R., Meynet, G., et al. 2006, A\&A, 449, L27 Chiappini, C., Ekström, S., Meynet, G., et al. 2008, A\&A, 479, L9 Dearborn, D. S. P., Lattanzio, J. C., \& Eggleton, P. P. 2006, ApJ, 639, 405 Decressin, T., Meynet, G., Charbonnel, C., Prantzos, N., \& Ekström, S. 2007, A\&A, 464, 1029

Denissenkov, P. A., \& Denissenkova, S. N. 1990, Sov. Astron. Lett., 16, 275 Denissenkov, P. A., \& Herwig, F. 2003, ApJ, 590, L99

Ekström, S., Meynet, G., Chiappini, C., Hirschi, R., \& Maeder, A. 2008, A\&A, 489, 685

Freytag, B., Steffen, M., \& Dorch, B. 2002, Astron. Nachr., 323, 213

Garcia-Berro, E., Ritossa, C., \& Iben, I. J. 1997, ApJ, 485, 765

Gil-Pons, P., Suda, T., Fujimoto, M. Y., \& García-Berro, E. 2005, A\&A, 433, 1037

Gil-Pons, P., Gutiérrez, J., \& García-Berro, E. 2007, A\&A, 464, 667

Gratton, R. G. 2007, in From Stars to Galaxies: Building the Pieces to Build Up the Universe, ed. A. Vallenari, R. Tantalo, L. Portinari, \& A. Moretti, ASP Conf. Ser., 374, 147

Grevesse, N., Noels, A., \& Sauval, A. J. 1996, in Cosmic Abundances, ed. S. S. Holt, \& G. Sonneborn, ASP Conf. Ser., 99, 117

Herwig, F., Bloecker, T., Schoenberner, D., \& El Eid, M. 1997, A\&A, 324, L81 Herwig, F., Freytag, B., Hueckstaedt, R. M., \& Timmes, F. X. 2006, ApJ, 642, 1057

Iben, I. J., Ritossa, C., \& Garcia-Berro, E. 1997, ApJ, 489, 772

Iliadis, C., Champagne, A., José, J., Starrfield, S., \& Tupper, P. 2002, ApJS, 142, 105 
Iliadis, C., D’Auria, J. M., Starrfield, S., Thompson, W. J., \& Wiescher, M. 2001, ApJS, 134, 151

Izzard, R. G., Lugaro, M., Karakas, A. I., Iliadis, C., \& van Raai, M. 2007, A\&A, 466, 641

Karakas, A. I., \& Lattanzio, J. C. 2003, PASA, 20, 393

Kepler, S. O., Kleinman, S. J., Nitta, A., et al. 2007, MNRAS, 375, 1315

Kudryashov, A. D., \& Tutukov, A. V. 1988, Astronomicheskij Tsirkulyar, 1525, 11

Lattanzio, J. C. 1992, PASA, 10, 120

Liebert, J., Bergeron, P., \& Holberg, J. B. 2005, ApJS, 156, 47

Marigo, P., Bressan, A., \& Chiosi, C. 1998, A\&A, 331, 564

Meakin, C. A., \& Arnett, D. 2007, ApJ, 667, 448

Mocák, M., Müller, E., Weiss, A., \& Kifonidis, K. 2009, A\&A, 501, 659

Mowlavi, N. 1995, Ph. D. Thesis, Université Libre de Bruxelles, Belgium

Należyty, M., \& Madej, J. 2004, A\&A, 420, 507

Nomoto, K. 1984, ApJ, 277, 791

Paczyński, B. 1970, Acta Astron., 20, 47

Palacios, A., \& Brun, A. S. 2008, in ed. L. Deng, \& K. L. Chan, IAU Symp., 252, 175

Poelarends, A. J. T., Herwig, F., Langer, N., \& Heger, A. 2008, ApJ, 675, 614

Pumo, M. L., D’Antona, F., \& Ventura, P. 2008, ApJ, 672, L25

Raiteri, C. M., Busso, M., Picchio, G., \& Gallino, R. 1991, ApJ, 371, 665

Refsdal, S., \& Weigert, A. 1970, A\&A, 6, 426

Ritossa, C., Garcia-Berro, E., \& Iben, I. J. 1996, ApJ, 460, 489
Ritossa, C., García-Berro, E., \& Iben, I. J. 1999, ApJ, 515, 381

Romano, D., Matteucci, F., Molaro, P., \& Bonifacio, P. 1999, A\&A, 352, 117

Romano, D., Matteucci, F., Ventura, P., \& D'Antona, F. 2001, A\&A, 374, 646

Sackmann, I.-J., \& Boothroyd, A. I. 1991, ApJ, 366, 529

Schwarzschild, M., \& Härm, R. 1965, ApJ, 142, 855

Siess, L. 2006, A\&A, 448, 717

Siess, L. 2007, A\&A, 476, 893

Siess, L. 2009, A\&A, 497, 463

Siess, L., \& Arnould, M. 2008, A\&A, 489, 395

Stoesz, J. A., \& Herwig, F. 2003, MNRAS, 340, 763

Travaglio, C., Randich, S., Galli, D., et al. 2001, ApJ, 559, 909

Tuchman, Y., Glasner, A., \& Barkat, Z. 1983, ApJ, 268, 356

Vassiliadis, E., \& Wood, P. R. 1993, ApJ, 413, 641

Ventura, P., \& D'Antona, F. 2005a, A\&A, 431, 279

Ventura, P., \& D'Antona, F. 2005b, A\&A, 439, 1075

Ventura, P., \& D'Antona, F. 2008, A\&A, 479, 805

Ventura, P., D’Antona, F., Mazzitelli, I., \& Gratton, R. 2001, ApJ, 550, L65

Ventura, P., D'Antona, F., \& Mazzitelli, I. 2002, A\&A, 393, 215

Wagenhuber, J., \& Groenewegen, M. A. T. 1998, A\&A, 340, 183

Wagenhuber, J., \& Weiss, A. 1994, A\&A, 290, 807

Woodward, P. R., Porter, D. H., \& Jacobs, M. 2003, in 3D Stellar Evolution, ed.

S. Turcotte, S. C. Keller, \& R. M. Cavallo, ASP Conf. Ser., 293, 45

Yoon, S.-C., Langer, N., \& van der Sluys, M. 2004, A\&A, 425, 207 